\title{
L-Py: an L-system simulation framework for modeling plant architecture development based on a dynamic language
}

\author{
Frédéric Boudon ${ }^{1}$, Christophe Pradal ${ }^{1}$, Thomas Cokelaer ${ }^{2}$, Przemyslaw Prusinkiewicz ${ }^{3}$ and \\ Christophe Godin ${ }^{2}$.
}

${ }^{1}$ CIRAD, Virtual Plants INRIA Team, Montpellier, France

2 INRIA, Virtual Plants INRIA Team, Montpellier, France

${ }^{3}$ Department of Computer Science, University of Calgary, Calgary, AB, Canada

\section{Edited by:}

Basil Nikolau, lowa State University, USA

\section{Reviewed by:}

Roeland Merks, Centrum Wiskunde \& Informatica, Netherlands

Haiquan Li, National University of

Singapore, Singapore

*Correspondence:

Frédéric Boudon and

Christophe Godin, INRIA Team Virtual

Plants, UMR AGAP, TA A-108/02,

Avenue Agropolis, 34398 Montpellier

Cedex 5, France.

e-mail: frederic.boudon@cirad.fr;

christophe.godin@inria.fr
The study of plant development requires increasingly powerful modeling tools to help understand and simulate the growth and functioning of plants. In the last decade, the formalism of L-systems has emerged as a major paradigm for modeling plant development. Previous implementations of this formalism were made based on static languages, i.e., languages that require explicit definition of variable types before using them. These languages are often efficient but involve quite a lot of syntactic overhead, thus restricting the flexibility of use for modelers. In this work, we present an adaptation of L-systems to the Python language, a popular and powerful open-license dynamic language. We show that the use of dynamic language properties makes it possible to enhance the development of plant growth models: (i) by keeping a simple syntax while allowing for high-level programming constructs, (ii) by making code execution easy and avoiding compilation overhead, (iii) by allowing a high-level of model reusability and the building of complex modular models, and (iv) by providing powerful solutions to integrate MTG data-structures (that are a common way to represent plants at several scales) into L-systems and thus enabling to use a wide spectrum of computer tools based on MTGs developed for plant architecture. We then illustrate the use of L-Py in real applications to build complex models or to teach plant modeling in the classroom.

Keywords: L-system, Python language, plant modeling, MTG, development, environment, FSPM

\section{INTRODUCTION}

In the last two decades, the study of plant functioning and development has been accompanied and supported by the development of a new family of models called functional-structural models (FSPMs, Sievänen et al., 1997; Godin and Sinoquet, 2005; Hanan and Prusinkiewicz, 2008). These computational models use 3D representations of plant architecture to simulate different types of physical, physiological, or ecophysiological processes in plants, and make it possible to assess the effects of these processes on plant functioning, development, and form.

The formalism of L-systems has emerged as the major paradigm for constructing FSPMs (c.f. FSPM Special Issue, 2005, 2008, 2011). Introduced in the late 1960s by A. Lindenmayer as a formalism for describing developmental processes in biology (Lindenmayer, 1968), L-systems proved well suited to describe models of plant development (Prusinkiewicz and Lindenmayer, 1990; Prusinkiewicz, 1998, 1999). In L-systems, the plant is represented by a bracketed string, whose elements, called modules, represent the plant's components (metamers, meristems, flowers, etc.). Modules consist of a symbolic name and an optional set of parameters. Modules with the same name represent the same type of component (e.g., I for internode, $\mathrm{M}$ for meristem, etc.). A set of rules (also called productions) then defines how each module transforms over time. In particular, a module can produce one or more new modules, thus giving a possibility of adding new components to the structure. Brackets are used to delimit the branches. When using L-systems, the modeler designs a set of L-system rules which, when applied step after step to the initial string (representing the initial state of the plant), will simulate its development.

During the past 20 years, several implementations of L-systems have been designed. The main ones used in plant modeling have been cpfg (Prusinkiewicz and Lindenmayer, 1990; Hanan, 1992; Prusinkiewicz et al., 1999a), lpfg (Prusinkiewicz et al., 2007; Karwowski and Prusinkiewicz, 2003), and XL (Kniemeyer and Kurth, 2008). Cpfg introduced a dedicated modeling language, in which L-system rules are written using a mathematical notation based on formal language theory. This notation is extended with C-like statements for specifying changes in parameters values. In the early 2000s, $c p f g$ was completely reengineered to address the needs of building more complex functionalstructural models. This gave rise to a new modeling program $l p f g$ and the modeling language $L+C . L+C$ extends $\mathrm{C}++$ with the notion of L-system productions. $X L$ relies on a similar approach, but is based on a different support language, Java, usually considered as a bit less efficient than $\mathrm{C}++$ but which offers at no cost portability between the different operating systems on which it runs. $X L$ manipulates dynamic structures made of modules that fully support object-oriented definition and extends the L-systems paradigm by making it possible to define 
production rules on structures more general than trees, such as graphs.

Despite the language difference, both $L+C$ and $X L$, share the common feature of being based on languages that are statically typed. By making it mandatory to define the exact type of variables that are manipulated in every part of the programs, statically typed languages can optimize the handling of data-structures, efficiency of computation, and early detection of errors (at compilation time, before execution). On the other hand, they constrain the user to strictly respect typing rules during programming, which involves high-level of programming expertize for users and, as a consequence, requires a steep learning curve (Ousterhout, 1998; Prechelt, 2000; Tratt, 2009). In contrast, dynamic languages are less exigent and do not require the specification of variable types in the code. They do manipulate types, but the correctness of variable types in expressions is mainly checked during execution. This involves an extra burden in program execution, resulting in a loss of overall efficiency with respect to statically typed languages, as many optimization schemes cannot be applied. On the positive side, the programming is more intuitive, the syntax is less austere, and the learning curve is much more shallow than for static languages (Tratt, 2009). Ousterhout (1998) illustrates this difference by pointing out that a typical statement in a dynamic language is equivalent to 100-1000 elementary instructions of the target machine while, in similar conditions, a typical statement in a static language corresponds to 1-10 elementary instructions. A higher level of abstraction is thus enabled by dynamic languages. Consequently, dynamic languages are frequently used as scripting languages, i.e., languages that allow fast prototyping both by fostering interactivity between programmers and their programs during development and by making it possible to glue together macroscopic software components easily.

The difference between static and dynamic languages can appear at first sight to be of a technical nature and of little interest to biologists. However, we suggest here that the use of dynamic languages is particularly well adapted to the building of simulation systems in developmental biology. In many modeling applications, the advantages of dynamic languages over static ones make the former an attractive choice despite their relative lower computational efficiency. They are more intuitive for users with a limited background in computer science, while offering recent, powerful object-oriented programming constructs for more computationally oriented users.

In this work, we explored the adaptation of dynamic languages to the modeling of plant growth. For this, we designed a new open-source L-system-based modeling environment based on Python, a popular and powerful dynamic language. An overview of the resulting language, $\mathrm{L}-\mathrm{Py}$, and its programming environment is presented in Section "L-Py Overview." Then we describe how L-Py can be used to model plant development at several scales. For topology, we extend classical L-strings to represent MTGs (formalism to represent the multiscale nature of plants), which makes it possible to seamlessly integrate a wide set of model components and tools already designed for MTGs into L-Py programs. For geometry, new primitives have been introduced in the language to describe plant components in a high-level manner and at different scales. Finally, we illustrate the use of L-Py in real modeling applications, composed of multiple modeling components and for developing training programs on modeling in the classroom (see Example of FSPM Applications in L-Py). All the code excerpts given in the paper are actual L-Py code. The corresponding example files can be downloaded together with the L-Py software through the OpenAlea distribution (http://openalea.gforge.inria.fr).

\section{L-Py OVERVIEW}

Embedding L-systems into a dynamic language such as Python has a number of consequences on the language syntax, its interpreter, the programming environment, and the openness of the system (i.e., its ability to interact with external components).

\section{A SIMPLE SYNTAX OWING TO DYNAMIC TYPING}

To integrate L-systems into Python, we followed a methodology similar to that used by Karwowski and Prusinkiewicz (2003) to design and implement $L+C$. L-system constructs were thus added to the syntax of Python, following the syntax of $c p f g$ and $L+C$ as closely as possible for compatibility between $L+C$ and L-Py. However, some constructs inherit specifically from the Python language syntax. Compared to $L+C$, the L-Py syntax is simplified by avoiding type declaration of parameters and variables. A simple example of L-Py code is given below.

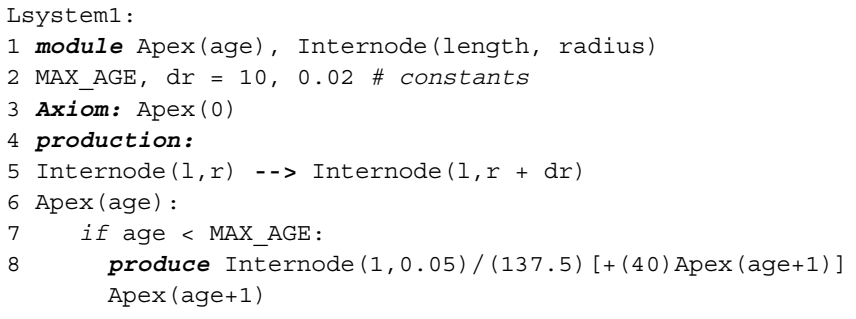

The main components of this code are as follows.

\section{Modules}

In L-systems, plant structures are decomposed into physical units called modules. The set of modules forms a branching structure that is represented by a bracketed string of modules (Lindenmayer, 1968; Prusinkiewicz and Lindenmayer, 1990; Hanan, 1992), here that we call here L-string. By default, any character can be a valid module name. Modules containing more than one character must be declared. Module parameters may also be declared to make explicit their role (although this is not mandatory), e.g., Apex (age) may represent an apex characterized by its age, as in line 1 of the above example. In this case, if Apex is found in a string, it will be interpreted as one module instead of four different modules, namely $\mathrm{A}, \mathrm{p}, \mathrm{e}$, and $\mathrm{x}$. Module parameters (such as age) are Python objects and can be of any type.

\section{Axiom}

A specific string of modules, called the axiom, defines the initial state of the simulation. This string is declared by using the 
keyword Axiom. In the example, the axiom is a string made of a single module Apex ( 0 ), representing the initial structure of the plant.

\section{Rules}

The keyword production indicates the beginning of rule declarations. As in $c p f g$, rules may have the syntax:

Predecessor --> Successor

or, more generally

LeftContext < Predecessor > RightContext --> Successor

where Predecessor and Successor are strings of modules, and both LeftContext and RightContext are optional strings of modules. The rules can be expressed using two conventions. Simple rules can be written in a compact mathematical style similar to cpfg (Prusinkiewicz and Lindenmayer, 1990; Hanan, 1992), e.g., line 5. Alternatively, for more complex rules, successor specifications are declared as in $L+C$ using the produce statement (instead of the arrow - - >) embedded into regular Python code, e.g., line 8. In this case, predecessors are separated from the right-hand side of the rules by a colon (line 6) consistently with the syntax of Python functions. For example, the rule of lines (6-8) replaces every Apex with parameter age inferior to MAX_AGE (line 7) with an Internode module followed by a lateral Apex and the main Apex. Lateral Apex is included in brackets. Two geometric symbols, / and + , make it possible to specify phyllotactic and insertion angles for the interpretation, respectively. The strings generated by this simple L-system in the first three simulation steps (starting with the axiom $w_{0}$ ) will be:

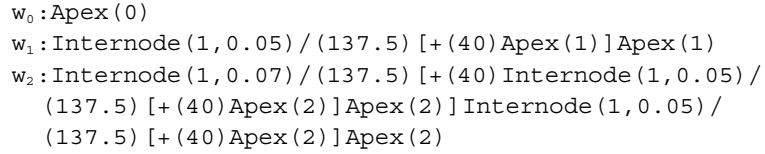

A visual representation of these strings is given in Figure 1. In summary, the syntax of L-Py is largely compatible with both $c p f g$ and $L+C$ to facilitate code porting between these languages. See Appendix for additional description of L-Py syntax.

\section{A FLEXIBLE INTERPRETER BASED ON DYNAMIC EVALUATION}

The L-Py interpreter makes it possible to execute L-Py expressions. The first phase consists of compiling the code of a model into bytecode that can be executed by Python (more precisely by the Python virtual machine). For this, L-Py code is first translated into pure Python code by the L-Py language parser which also generates some internal structures for the interpreter. During this process, the predecessor of each rule is stored in a dedicated data-structure, and the rule is transformed into a corresponding Python function. Due to the runtime evaluation ability of dynamic languages (Tratt, 2009), evaluation of the Python code was easy to implement in L-Py.

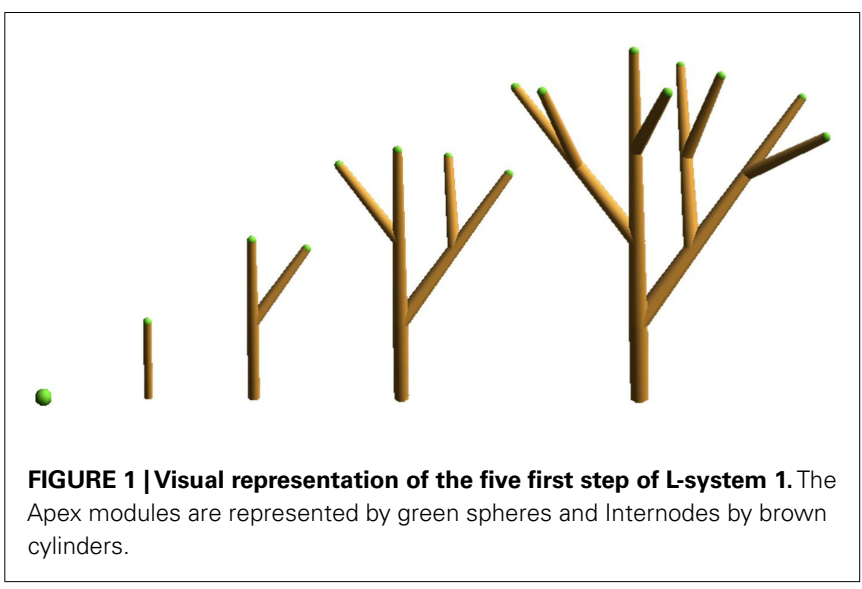

Following the definition of L-systems, the L-Py interpreter applies productions to the current string of modules (called Lstring) as many times as specified by the user. In each derivation step, a new L-string is computed by transforming the L-string resulting from the previous step (or the axiom in the first step). To this end, the interpreter parses the L-string and looks for modules matching production predecessors. In L-Py, modules may have a dynamic number of parameters, therefore both the name of a module and its actual number of parameters are taken into account while matching a module in the string to a production predecessor, as in cpfg (Prusinkiewicz and Lindenmayer, 1990; Hanan, 1992). If a match is found, a Python function corresponding to the identified production is called and the string of modules returned by this function (typically found after a produce statement) is appended to the currently constructed L-string. If several matches are found, the first one is used.

In addition to applying productions, L-Py can perform a geometric interpretation of the new L-string in each derivation step upon request. The blocks of rules used for this purpose are preceded by the keyword interpretation. Interpretation rules that match the new L-string modules transform modules of the new L-string into predefined symbols that can be geometrically interpreted by a Logo-style turtle (Prusinkiewicz, 1986). To this end, L-Py was strongly coupled with PlantGL, a Python-based graphic library for plant modeling (Pradal et al., 2009), which provides many high-level graphic and turtle geometry primitives (see also High-Level Constructs for the Control of Turtle Geometry). Given an L-string, the turtle builds a graphic scene that can be displayed with the PlantGL 3D viewer (Pradal et al., 2009).

\section{A POWERFUL OPEN SYSTEM ENABLED BY LANGUAGE INTROSPECTION}

Thanks to the embedding in a dynamic language, L-Py functionalities can be easily accessed either through a graphical interface or directly using Python commands. This enables users to control L-Py programs in three different manners.

\section{A complete integrated development environment}

The $c p f g$ and $l p f g$ simulators are embedded in modeling packages vlab (Mercer et al., 1990; Federl and Prusinkiewicz, 1999) and L-studio (Prusinkiewicz et al., 1999b; Prusinkiewicz, 2004), which 
provide graphical editors of model attributes such as colors, optical properties of materials and graphically defined functions, curves, and surfaces. Likewise, the L-Py interpreter has been embedded into an integrated development environment (IDE) that includes code and visual parameter editors. The code editor incorporates dedicated syntax highlighting. When an error occurs, the corresponding line is automatically highlighted, facilitating the debugging of the model. Visual editors make it possible to interactively edit scalars, curves, patches, materials, and graphically defined functions. Names of these objects can be defined in the editors and are used to manipulate the objects in the L-Py code of the models. Similarly to L-studio, L-Py also contains a continuous modeling mode in which interactions with parameters are immediately propagated and visualization of the model automatically updated. This facilitates interaction with the model during simulations.

In addition to the visualization of the graphical interpretation of the model, an interactive exploration of the computed L-string is of great help to characterize and validate a model. To achieve this, a standard Python shell has been integrated in the development environment with primitives to access the computed L-strings and other variables. This allows inspection and a posteriori processing of the structure. A similar approach can be found in the GroImp platform, which includes a home-made interactive interpreter, $X L$ (Kniemeyer and Kurth, 2008).

The L-Py IDE is also inspired by programming environments such as Visual Studio (Microsoft Corporation, 2011). Its configurable interface using dockable panel widgets (see Figure 2) makes it possible to easily customize the working environment and its advanced development tools (profiler and debugger, etc.). The LPy IDE incorporates two tools that help optimize models. First, a debugger shows the user the successive rule applications that occur during a derivation step with actual parameters and global variable values. The debugging can be constrained to detect the application of particular rules only. Second, a profiler provides the user with a detailed report of the time spent in each rule and function, and makes it possible to identify bottlenecks in the execution. The creation of these tools was facilitated by the introspection property of dynamic languages (introspection is the ability of a program to explore and possibly modify its own code). For instance, access at

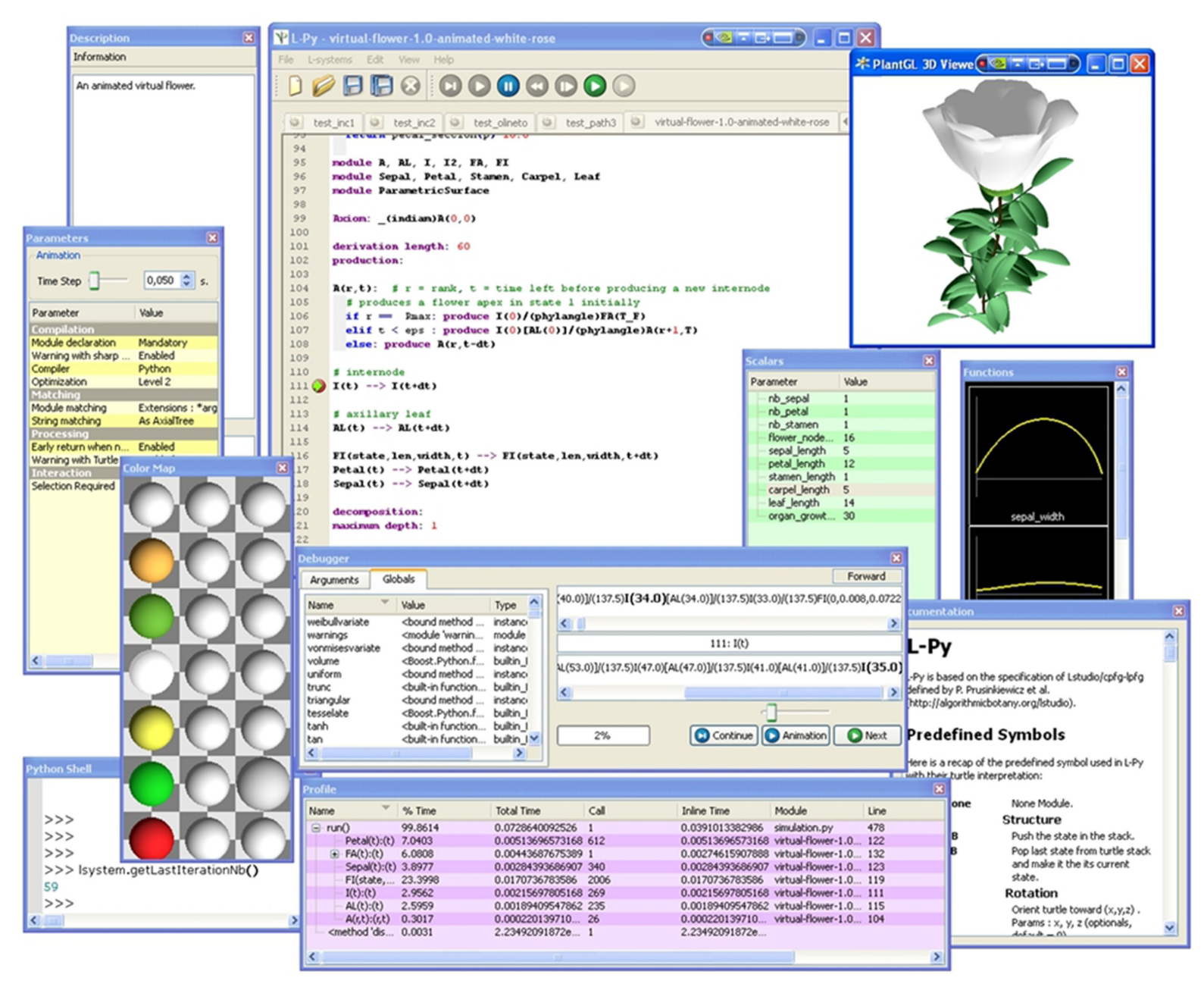

FIGURE 2 |The L-Py IDE with its utility windows for parameter edition. 
runtime to the names and values of the different variables involved in a procedure is simple in a dynamic language and facilitates, for instance, the creation of the debugger.

\section{L-Py as a component library: controlling L-systems execution from Python}

L-Py has been developed as a C++ library embedded in Python that can thus be integrated into any Python-compatible application. For this, the L-Py library defines a number of structures (module, L-string, rule, L-system, etc.) that can be accessed from Python in an object-oriented manner. As a result, an L-system model can easily be manipulated by an external process. Such a process typically creates an L-system object, executes it for a number of specified steps, possibly changes its parameters, resumes the execution, and finally gets the computed L-string. In this way, L-Py can be encapsulated as a simple component of a more complex modeling pipeline that integrates other components, possibly using formalisms different from L-systems.

The central issue of such an encapsulation strategy is to control the execution of L-Py models. An L-Py program contains global variables, functions, rules, and configuration/execution variables such as the number of derivation steps. From an external process, a user may want to access and change any of these elements. In L-Py, this can be done by using the Python/L-Py introspection mechanism or by using dedicated primitives implemented in L-Py. For instance, to explore a large parameter space for a given L-system, a user may want to vary parameters (e.g., global variables) of an L-system model after each execution. Such exploration cannot be made easily manually. The use of the introspection property of L-Py makes it possible to resolve this issue elegantly: a process that uses L-Py as a component can inquire about the internal variables of the L-Py model (global and configuration/execution variable) and directly access and modify them in memory. Global variables of the model become automatically and dynamically attributes of the L-system structure that contains the model, and can be modified as easily as any attribute of a Python object. Execution variables can also be modified with predefined L-Py functions. For example, to overwrite the axiom of an existing L-system object, an L-string can be built from a specified string of characters with the Lstring construct and then used to overwrite the contents of the L-system axiom. More generally, L-strings can be built from modules containing objects of any complex type as parameter values. Thanks to the dynamic typing of the language, parameters of any type can be introduced into the L-string and passed to an L-system. For example, the following program creates and runs iteratively Lsystem 1 using an increasing value of the global variable $d r$, incremented externally in each step.

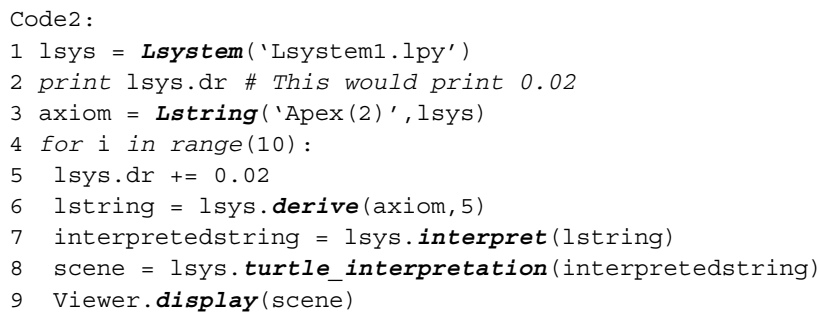

Line 1 reads in and creates an L-system structure from the Lsystem 1 code. The second line requests and prints the value of the internal variable dr from the model 1sys. Note that the global variables such as dr are automatically considered as attributes of the L-system object. Line 3 creates an L-system string from a text string, using 1sys module definition to interpret correctly the module names. This L-string will be used subsequently as the L-system axiom. Line 4 initiates a loop that will perform parameter space exploration for ten values of the dr parameter. At each iteration step, Line 5 changes the value of $\mathrm{dr}$ before performing 5 Lsystem derivation steps starting from the axiom (line 6) and assigns the resulting string to lstring. Note that in this case, only the production rules and the global variables of the L-system are used in the object, while the initial string (axiom) can be considered as a variable (notion of L-system scheme, Herman and Rozenberg, 1975). The function derive can also be called with no argument and will use in this case the values of the axiom and the number of derivation to perform declared in the L-system. Finally, lines 7-9 interpret the resulting string geometrically using the turtle interpretation, and display the result in the viewer. These lines can also be summarized into one (more efficient) command lsys.plot (lstring). However, they are given here to show that a user can finely control the execution of an L-system. Importantly, entire functions and productions of complex models can be changed similarly to the simple variable $d r$ in the above example.

Creation and manipulation of an L-system object have also been encapsulated into OpenAlea (Pradal et al., 2008) as computational nodes. Similarly to the example provided here, these nodes can be created with L-Py code and parameterized with a dictionary containing the names and new values of the variables. Examples of such use are given in Section "L-Py as Growth Component for Simulating an FSPM."

\section{The L-Py introspection mechanism: controlling L-system execution from L-Py programs}

L-Py makes it also possible to control its execution, variables, and rules from within L-Py models. For instance, the ExecutionContext object is accessible through the execContext () function and makes it possible to ask and modify the values of the L-Py model configuration/execution variables. These variables control the number of derivation steps, the currently used group, etc. (a complete list is given in the online help; see also Standard L-Systems Features of L-Py in Appendix). After each iteration modelers also have access, through the EndEach function, to the resulting L-string and the corresponding scene graph. This enables global post-processing of the modeled structure using regular Python code and external modules from within a model. In this way, an L-Py model can also act as an integrative framework for different modeling components (see for instance section "Minimizing Measurements in 3D Plant Architecture Reconstruction"). For example,

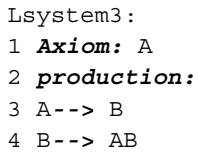

produces a sequence of strings whose lengths follow the Fibonacci series. It can also be shown that the string produced in step $t$ is the 
concatenation of the strings produced in steps $t-2$ and $t-1$. Checking visually these properties with L-Py is easy and requires only adding two lines of code:

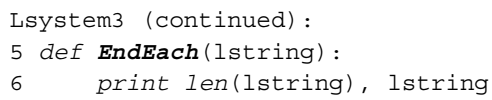

These lines print the length and the value of the current L-string and are called after each derivation step. The output exhibits the Fibonacci properties of the model:

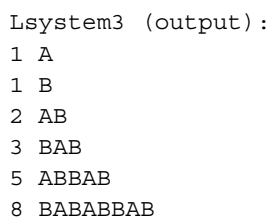

Modification to the string can be made in a similar way from within a L-Py model. Likewise, thanks to the dynamic language evaluation, it is possible to add or remove a rule during the execution of an L-system.

\section{MODULAR L-SYSTEMS IN L-Py}

Code modularity is the key to build complex and reusable models. In our context, this raises the question of building reusable blocks based on L-systems that can subsequently be assembled. Modularity of the model may result from the decomposition of the structure into components such as internodes or leaves, which can be processed and defined independently (Hanan, 1992; Godin et al., 1998; Prusinkiewicz et al., 1999a) or from the decomposition of the model into of component aspects such as growth, photosynthesis, and hormone transport (Cieslak et al., 2011). In this section, we discuss the support for decomposition into aspects, offered by L-Py.

One technique supporting such decomposition was proposed by Federl and Prusinkiewicz (2004), based on the concept of controlled derivations in L-systems. Different groups of production rules are identified by group Ids. Only one group is active in any derivation step. However, another group may be activated in the next derivation step, and so on. The selection of the appropriate group can be conveniently made before each derivation step using the StartEach statement. Within a static language, however, parameters of the modules are declared at the beginning of the model and their declaration should take into account all parameters required for all groups. This limits the independence of the groups and thus the modularity of the composite L-system.

More recently, Cieslak et al. (2011) proposed another strategy to develop modular L-systems, based on the use of separate modules to represent different aspects of the model. These modules can be combined such that one organ of the plant is represented by a list of modules, each reflecting a different aspect of the model. The rules related to the different aspects are described in different groups and are invoked sequentially. While this makes it possible to have separate definition of each group with their own modules and rules, a given element is modeled in this approach with several modules, which blurs its identity.
In L-Py, we elaborated on the approach of Federl and Prusinkiewicz (2004) and extended it with the use of a dynamic language to reinforce the decoupling of the different L-systems to assemble. The goal is to combine several independent L-systems, typically written by different persons, in different files (say for example files A. Ipy, B. Ipy, C. Ipy). Each L-system can be considered as a processing unit dealing with an aspect of simulation, for example substance transport, branch mechanics, or growth. An order may have to be respected in the application of the corresponding L-systems, as some of them may update L-string parameters subsequently used by other L-systems. We assume that the different L-systems operate on the same or closely related sets of plant components, e.g., apices, growth units, or internodes. As similar components in different L-systems may be identified by different module names, a mapping between these names is defined in the third "translation" L-system. For instance, if the module representing an internode is named $I$ in L-system $A$ and $S$ in Lsystem $B$, a rule $I$ - - > S will be created in the translation $\mathrm{L}$-system A2B. Module parameters must also be translated. Detailed examples of how this is done are given in Section "Managing L-System Modularity" in Appendix.

Different L-systems and their translations can be chained by the programmer to make up a unique compound Lsystem. To easily handle such chaining, a generic Python class ComposedLsystem is provided in L-Py. It takes two arguments: a list of L-systems to be chained (including translation schemes) and a list of interpretation schemes to be chained. A sketch of a typical code that must be defined to combine different L-systems follows.

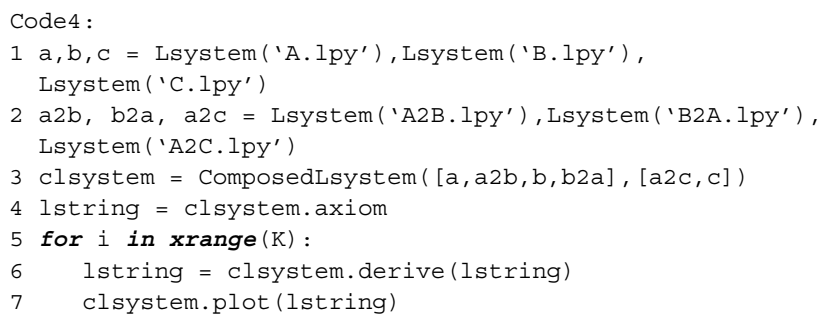

The first two lines create the different L-systems required for the model. The third line gathers them into a ComposedLsystem structure. As arguments, two lists of L-systems are given. The first list contains the set of L-systems responsible for the production rules and the second list for the interpretation, given in the order in which they should be called. In the first list, the target L-system of the last translation (the " $\mathrm{a}$ " in b2a in the example below) must be identical to the first L-system of this list, while in the second list, the source L-system of the first translation (" $a$ " in a $2 c$ ) must be identical to the last L-system of the first list (i.e., "a" also). The next four lines simply run and plot the ComposedLsystem for a number $\mathrm{K}$ of iterations as if it were a simple L-system. In this case the chaining of L-systems is controlled by the ComposedLsystem primitive, but the modeler still has the possibility to write code that calls each Lsystem one after another a number of times (for instance to handle different time units in the different L-systems).

This approach shows a great flexibility in assembling components by transforming L-strings expressed in the alphabet of 
one L-system to that of another one. This approach may be combined with sub-Lsystems or other aspect-oriented approaches into a completely flexible and modular L-system framework.

\section{MODELING PLANT GROWTH AT DIFFERENT SCALES IN L-Py}

The embedding of L-Py in a dynamic language has a number of consequences on the modeling possibilities themselves. Due to the non-strict typing system, connection with external modules and models is much simpler than with static languages. Here, we investigate the consequences of this language specificity to key aspects of plant modeling.

\section{SEAMLESSLY COMBINING L-SYSTEMS AND MTGs}

In the late 1990s, Godin and Caraglio (1998) introduced a formal model called Multiscale Tree Graph (MTG) to represent a wide range of plant architectures, at varying scales of description, in a flexible and unified way. Since then, MTGs have been used widely to encode various types of plant architectures at varying scales (e.g., Godin et al., 1999; Mündermann et al., 2005; Teobaldelli et al., 2008) and to analyze the resulting data with dedicated software such as AMAPmod (Godin and Guédon, 1997) and OpenAlea (Pradal et al., 2008). Today, in OpenAlea, MTG is the central datastructure that different modeling packages use as a standardized way to represent plants.

In order to exploit the large library of algorithms and models built for MTGs in L-Py, we designed bidirectional translation and mapping mechanisms between L-strings and MTGs. Conversion between these structures is possible as they both represent a particular type of labeled tree graphs. L-strings represent axial tree graphs (Prusinkiewicz and Lindenmayer, 1990) while MTGs may integrate tree graph descriptions at several scales (Godin and Caraglio, 1998). Fortunately, it has been shown that, similarly to simple tree graphs, multiscale tree graphs can be encoded as strings (Godin et al., 1999). L-strings corresponding to MTGs can be defined using this property (Godin et al., 1999; Ferraro and Godin, 2000).

In brief, let us first consider an L-string representing the tree graph at the most microscopic scale of the MTG (e.g., at the scale of internodes I), of Figure 3 (left) e.g.,

\section{I I $\left[\begin{array}{lll}I & I\end{array}\right]$ I...}

where each I represent a plant module (here an internode), and opening brackets indicate branching points. Modules representing more macroscopic nodes of the MTG, corresponding for example to growth units $U$ or to branching systems $S$, are inserted before the first microscopic module that composes them (Godin et al., 2005). The resulting string mixes modules at different scales. A small branching system $S$ composed of growth unit $U$ that can be decomposed as internodes $I$ is thus encoded as:

S U I I I [U I I] U I...

This defines a multiscale L-string associated with the MTG (see Figure 3). For the L-Py interpreter to recognize that modules in the string belong to different scales, the user must explicitly associate each module type with a scale using the keyword "scale" in the module type declaration of the L-Py program:

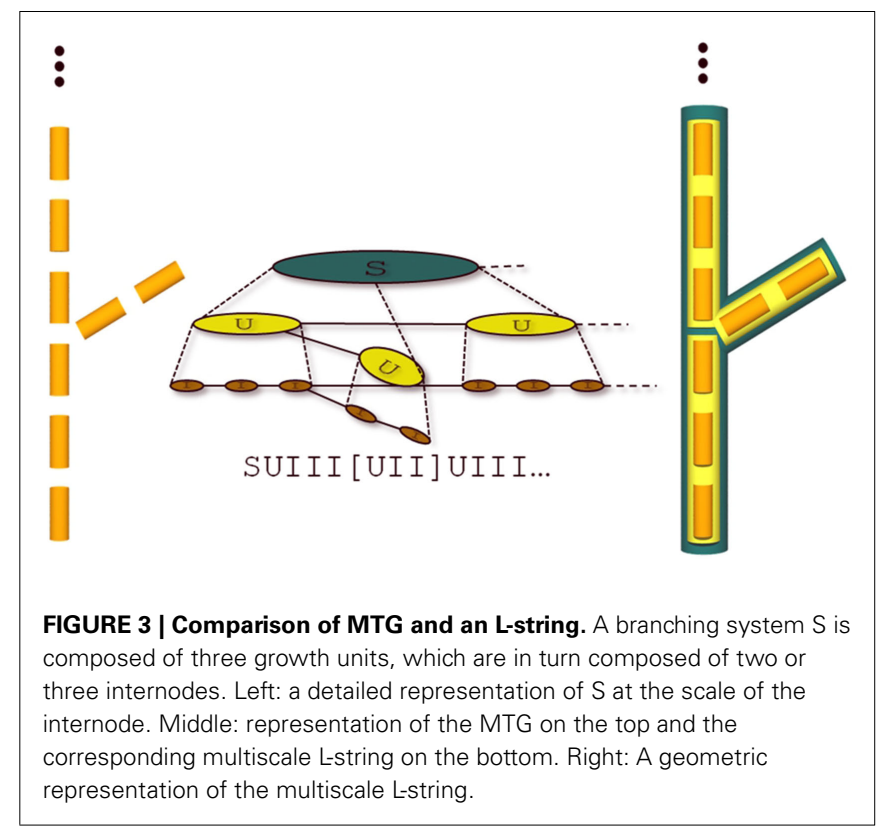

module $\mathrm{S}: \mathrm{scale}=0$

module U: scale $=1$

module I: scale $=2$

As in classical L-strings, modules in multiscale L-strings can have parameters of any type, including complex types and objects. The use of a dynamic language makes it possible to seamlessly convert L-strings into MTGs and, reciprocally, MTGs into L-strings. Indeed, in the conversion, L-string module parameters are automatically transformed into MTG node parameters (or vice versa) without the burden of duplicating parameters in memory or writing/reading data through exchange files.

Primitives to read/write and convert MTGs into L-strings (and reciprocally) have been designed and make it possible to manipulate MTGs directly within L-Py rules. These can be used for instance to initialize a simulation with a plant architecture measured experimentally:

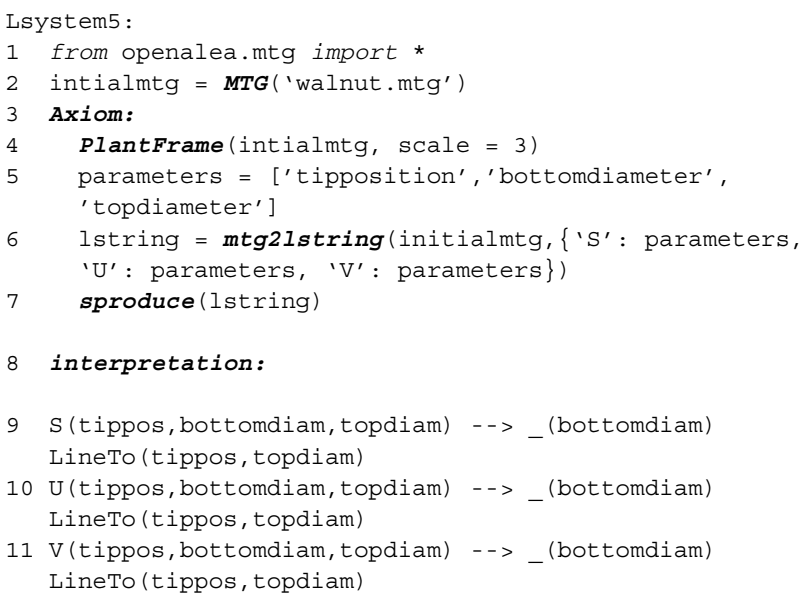

Axiom is now defined as a rule (lines 3-7) that produces a string (line 7 ). The MTG of a measured walnut tree (Juglans regia L.; 
Sinoquet et al., 1997) is first loaded (line 2, note that the functions for manipulating MTGs, such as MTG and PlantFrame used here, are independently provided by the MTG package of OpenAlea). This file contains the information related to the topology of the plant at three different scales (axis segments, axes, and plant). In addition, for some plant segments, it contains key information about their geometry, called "frame" information (this information was not systematically measured for all plant segments in the field). The frame information consists of the spatial location of the segment tip in a reference coordinate system originating at the basis of the plant, together with their bottom and top diameters. Based on the frame information available for some segments in the MTG, the Plant Frame function makes it possible to compute the frame information for all the plant segments where it is missing, using predefined inference rules (Godin et al., 1999). As a result, the frame attributes ('tip position', 'bottomdiameter' ,'topdiameter') of the segments of the MTG are updated with the computed information. The MTG is then transformed into a multiscale L-string (lines 5-6), where the different modules corresponding to the plant segments (here labeled $S, U, V$ ) are given parameters corresponding to their frame information in the MTG. Finally, the sproduce statement of line 7 produces the multiscale Lstring corresponding to the MTG (one can note the difference between produce and sproduce: produce creates a successor L-string from a list of modules, while sproduce creates a successor L-string from an already built L-string structure). The axiom defined by this string has thus been procedurally evaluated. Finally, to plot a graphical representation of the axiom, a simple interpretation rule is defined for each type of module (line 9-11) that uses the turtle to draw the complete plant structure by exploiting the frame data of the successive plant segments along the plant axes (Figure 9, left).

\section{HIGH-LEVEL CONSTRUCTS FOR THE CONTROL OF TURTLE GEOMETRY}

As illustrated in the previous sections, the use of a dynamic language such as Python favors the openness of the modeling language (i.e., its ability to be extended) and its simplicity of use by providing high-level constructs in the language. Both characteristics were considered as key guiding principles throughout the design of L-Py. In this section, we show how these principles were used to simplify the modeling of plant geometry by introducing new constructs to manipulate turtle geometry at high abstraction level.

\section{Custom geometric primitives for plant representation at different scales}

When representing plant architecture, most simulation systems use an explicit geometric representation of plant organs: internodes are represented by cylinders, leaves by small parametric surfaces, fruits by volumetric models, roots by generalized cylinders, etc. In recent years, however, abstract geometric models of plant organs have been introduced to represent plant architectures at more macroscopic scales in simulation models (e.g., Cescatti, 1997; Boudon, 2004; Pradal et al., 2009; Livny et al., 2011). These approaches are based on the use of either volume or envelope models that represent groups of organs instead of individual organs. Such models can be readily designed in L-Py thanks to the tight coupling with the PlantGL library (Pradal et al., 2009). A generic primitive, @g (geometry), allows the modeler to position any PlantGL model in space using the current turtle location and orientation. In this way, coarse geometric representations of plant architecture can be defined where parts of the tree crown are represented by parametric envelopes. The resulting architecture may then be used in conjunction with ecophysiological models that take a 3D scene as an input. Here we illustrate this possibility by computing the direct illumination of each crownlet in the plant using the Fractalysis library (Da Silva et al., 2008) from OpenAlea:

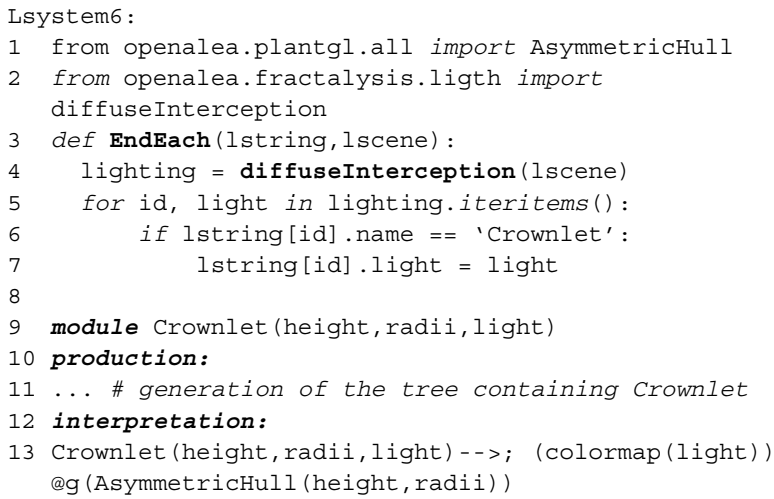

This L-system produces in each derivation step an L-string that is composed of modules Crownlet which are characterized by their height, maximum radii in four directions in the plane perpendicular to their main axis, and the total amount of light that they receive (defined in line 9). As explained in Section "The L-Py Introspection Mechanism: Controlling L-System Execution from L-Py Programs," the L-string produced at each step and the corresponding L-scene can be post-processed in the EndEach function. The L-scene is a set of PlantGL objects that were derived from the Lstring modules by the application of the interpretation rules (line 13). Each L-scene object thus contains an id corresponding to its associated module in the L-string (in L-Py, the L-scene ids simply correspond to the position of their associated module in the L-string). In the EndEach function, the amount of diffuse light intercepted by every module of the plant is evaluated by a call to the Fractalysis library (line 4). The diffuseInterception primitive is passed a PlantGL scene and returns a dictionary containing pairs composed of module ids and of the amount of light intercepted by that module. To import this information back into the string, an iteration over the dictionary is made in lines 5-7. For each module id, the module name is checked (line 6) to select only crownlet modules and their light parameter is updated (line 7). Figure 4 shows a representation of the L-scene after construction of a tree made of a branching system bearing crownlets represented by asymmetric hulls and colored according to the total amount of light intercepted by each crownlet.

\section{Seamless control of differential turtle geometry}

In particular modeling situations, one needs to instruct the turtle to follow a predefined curve in $3 \mathrm{D}$ space. This is required for example when one wants to control the shape of a branch using a predefined template shape. For this, we assume that a curve of 
length $\mathrm{L}$ is defined that represents the shape of a particular branch. In the $3 \mathrm{D}$ scene, at the position of the branch insertion on the parent branch, we then need to instruct the turtle to move along this curve from its current position. To achieve this, Prusinkiewicz et al. (2001) designed an algorithm to move the turtle in the 3D space based on differential geometry and using quantities such as local tangent, curvature, and step size. The following L-Py code gives a simple $2 \mathrm{D}$ version inspired from this algorithm.

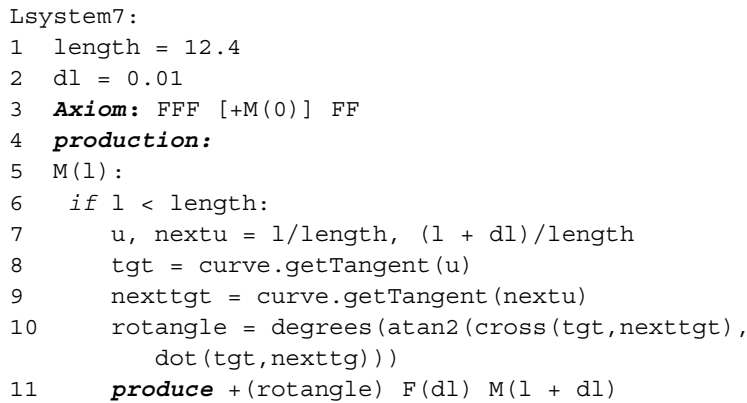

The axiom defines a parent branch made of five segments and bearing a branch on the third one (line 3 ). The branch is produced by incremental application of rule $\mathrm{M}(i)$ until length length is reached (lines 5-6). First, normalized linear abscissa of current and next points are computed (line 7), assuming that the turtle will make steps of constant size dl. This makes it possible to compute the angle by which the turtle should turn (lines 8-10) according to the reorientation of the tangent between these points before making the move (line 11). The recursive application of this rule produces the branch shape as specified by the PlantGL Curve2D object curve, defined elsewhere either graphically or procedurally (see section "A Complete Integrated Development Environment" and Figure 5B).

However, one can note that the corresponding code contains low-level instructions related to the computation of the local

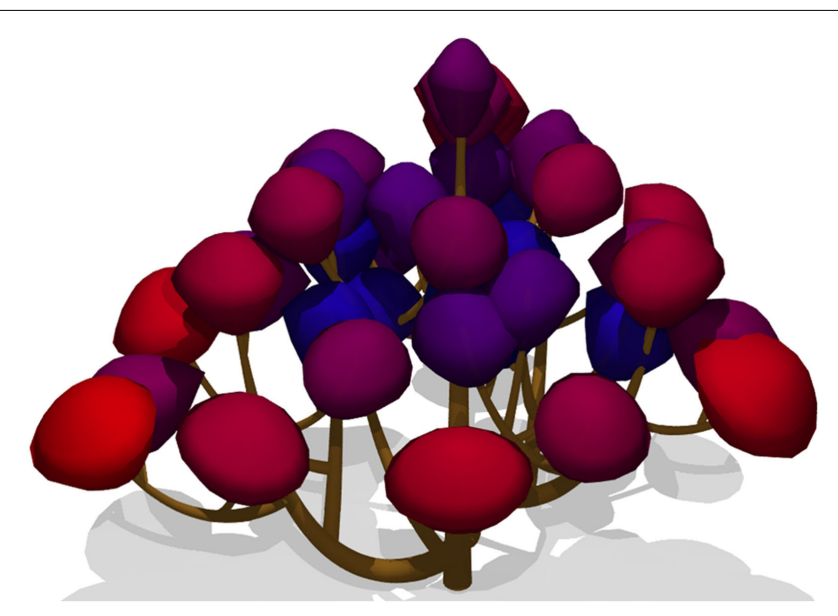

FIGURE 4 | Representation of a tree at the scale of crownlet using of the asymmetric hull primitive of PlantGL and with light interception computation using Fractalysis (Da Silva et al., 2008). curvature on the template curve (lines 7-10). This may obscure the overall code with instructions related to differential geometry management, which are not essential to the expression of the model itself. To alleviate this difficulty, we abstracted this differential geometry management by introducing the primitive SetGuide (curve, length), which instructs the turtle to follow the given curve until the total length of its moves reaches the prescribed value length. The algorithm used to control the turtle frame movement from the curve definition is inspired from Bloomenthal (1990) to control branch shape in a global to local manner (details of the SetGuide primitive are depicted in section "The SetGuide Primitive" in Appendix). Using this primitive, we can define the shape of a branch and keep clear the main L-system code:

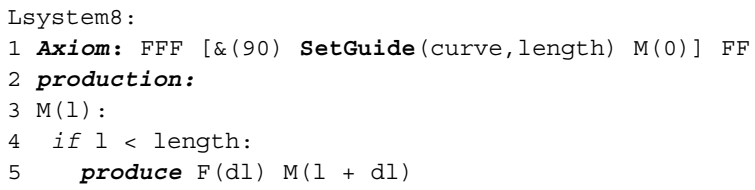

In the axiom (line 1), as soon as the turtle has been rotated to draw the branch, it is instructed to follow the curvature specified by the template curve curve (SetGuide primitive). The turtle is moved recursively forward following at each step the bends defined by curve, leading to the result depicted in Figure 5B. Note that if the SetGuide primitive is removed, the code is still valid L-Py code, where the turtle goes straight instead of following any curved trajectory (Figure 5A). In fact, SetGuide made it possible to completely separate the specification of the branch geometry from the specification of the topology.

This design pattern can be applied to a more complex branching system to control the shape of branches in a global to local manner. The following code illustrates the use of SetGuide to control the bending of a complete branching system recursively with a unique template curve.

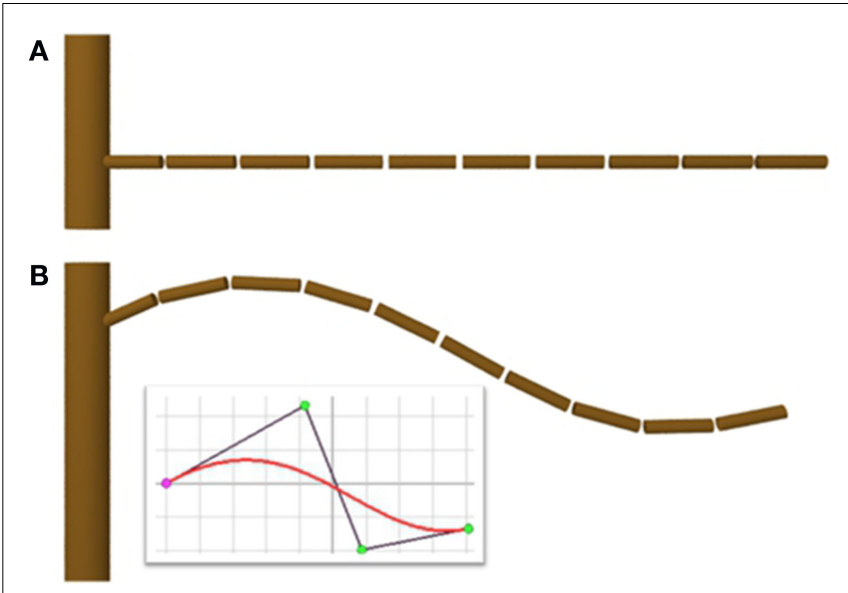

FIGURE 5 | Construction of the geometry without (A) and with (B) the SetGuide primitive. It takes as input a user-defined template curve (represented in the inserted window) and control curvature of turtle path. 


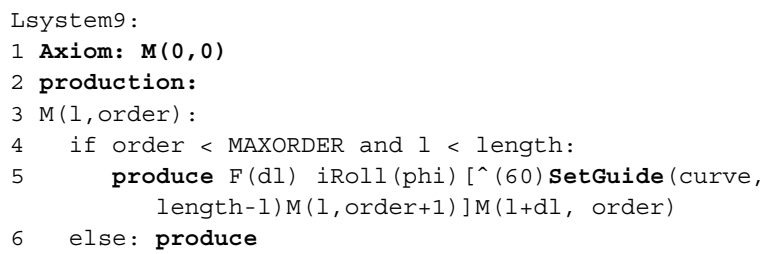

The apex M now has an additional parameter "order" to control the order of the branch. Apices whose order is greater than MAXORDER abort (line 4). The apex of order 0 is not prefixed by any SetGuide (line 1) and thus assumes a Euclidean space and develops a straight vertical trunk. By contrast, the branches built by lateral apices at order 1 and 2 are all prefixed by a Set Guide (line 5 ) and will then follow the specified template curve curve for the remaining length length-1. The resulting branching structure is illustrated in Figure 6.

\section{Modeling shape variation}

Plants architectures frequently show gradients in the shape of their organs. At different scales smooth variations of form and orientation may be observed: in petal shapes in flowers, in branch bending along a trunk, or in crownlet shapes and volumes in a tree crown (e.g., Bell, 1991; Barthélémy and Caraglio, 2007). Continuous variations in shapes may also arise throughout time due to growth and aging: leaves may unfold out of the bud for instance or fold due to a change in their water status, branches may change their shape due to interaction between gravity and growth, while trees may undergo deep shape metamorphosis throughout their lifespan (Hallé, 1978). Common to all these processes is the notion that a shape changes seamlessly (or "continuously") either in space or in time. Describing such changes is critical in models of plant architecture. In the context of L-systems, the importance of this phenomenon was recognized by Prusinkiewicz et al. (2001) who proposed to model attributes of plant architecture as functions of their location along the main axis (positional information).

Here again, the tight coupling between L-Py and PlantGL provides a powerful solution to address this issue. The ProfileInterpolation object of PlantGL makes it possible
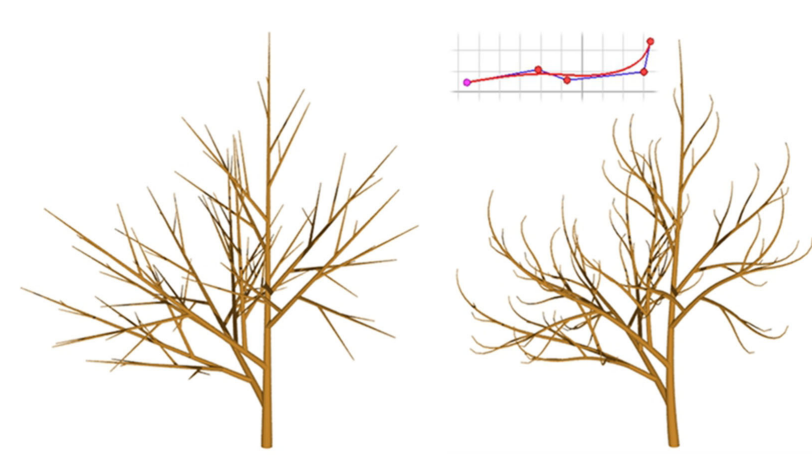

FIGURE 6 | Left: simple recursive structure with straight branches, Right: use of a template curve shown in the inset to define branch geometry. S-shaped branches produce a more realistic appearance and can be easily specified in L-PY. to smoothly interpolate between user-defined curves. The user specifies a set of keyframe curves at given index values. Then the ProfileInterpolation uses an interpolation scheme (e.g., the BSpline interpolation scheme; Piegl and Tiller, 1997) to compute intermediate curve values for any index between the extreme index values.

This function can be used for instance in combination with SetGuide to control the shape of axes in a branching system in a high-level manner. Using positional information (Prusinkiewicz et al., 2001) and ProfileInterpolation, we can compute for every position on the trunk of the plant a branch shape defined as a smoothly interpolated value between user-defined curves at different key altitudes on the trunk.

Let us modify for instance the previous L-system to control the shape of branches on the trunk according to a gradient of template curves.

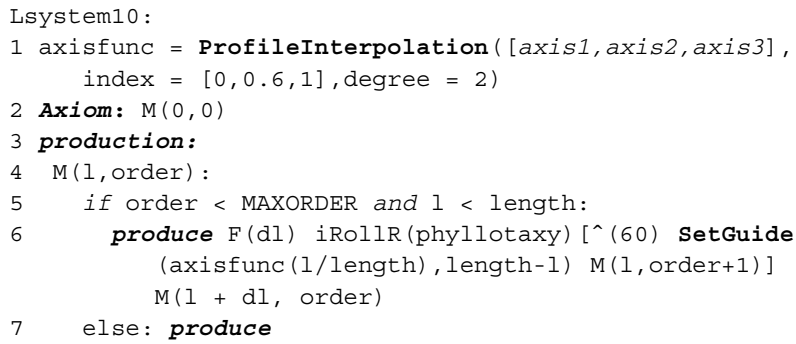

An interpolation scheme, using these reference curves is set up by specifying the normalized indexes corresponding to these curves, and the degree of interpolation (line 1). Then the SetGuide is set up to move on the axis curve defined for the normalized index $1 /$ length by the interpolation scheme. Figure 7 illustrates the result of this scheme applied to different sets of key curves.

This interpolation procedure can also be used to animate plant development in a flexible manner as illustrated by the example code below (Figure 8).
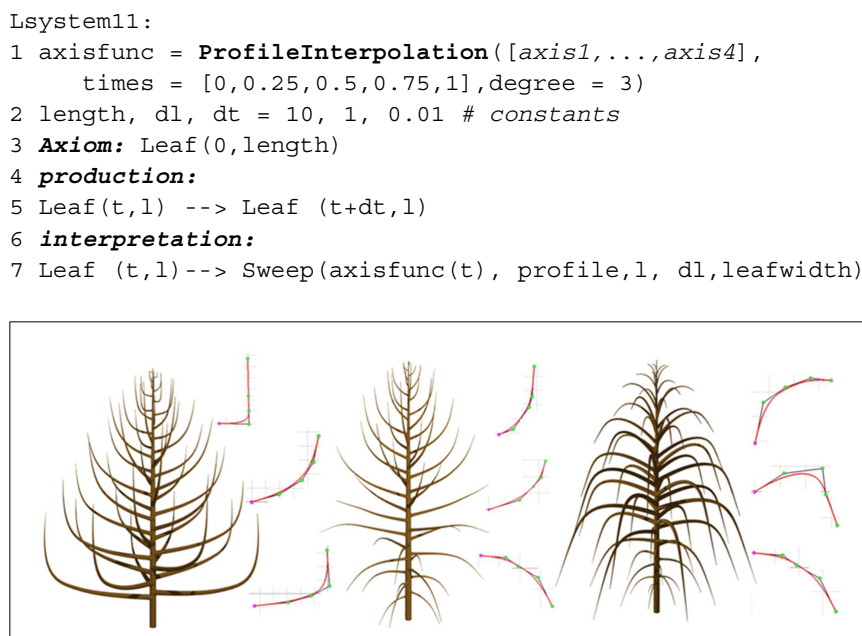

FIGURE 7 | Examples of structures whose branches shapes are defined as interpolation of three curves (shown on the right of each tree). Lengths of lateral branches are also dependant of the position of the branches on the trunk and is controlled with an extra graphical function. 


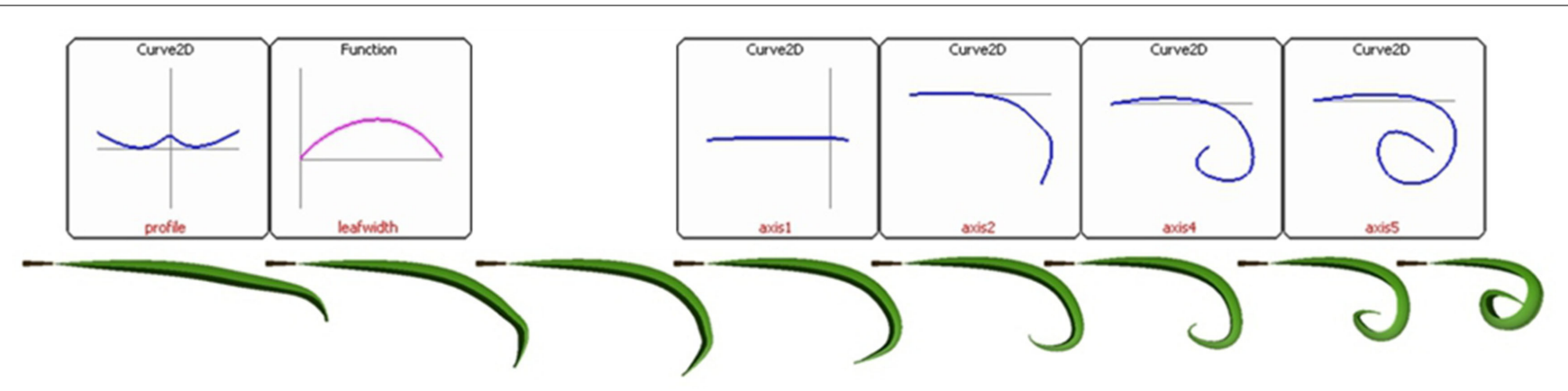

FIGURE 8 |Animating a rolling leaf (bottom) using graphically specified curves and functions (top). The first curve specifies the cross section of the leaf, the second specifies leaf width along the main axis of the leaf, and the remaining four curves specify the key shapes of the main axis of the leaf over time.

A sequence of keyframe curves defining the midrib of a leaf gradually rolling down were defined graphically by the user (Figure 8, top right). An interpolation scheme is set up using these reference curves by specifying their time points and the degree of interpolation (line 1). The L-system production simply advances the "age" $t$ of a module representing the leaf (line $5)$. Each application of this production is followed by an interpretation step (line 7). The produce statement in line 7 creates the leaf blade geometry. The leaf midrib is specified by the LPy built-in Sweep primitive that corresponds to an extension of generalized cylinders (Bloomenthal, 1985) to arbitrary contours, including non-closed contours. This primitive is itself defined on the basis of the SetGuide primitive. In the example code, the contour is specified by the Setsection primitive and defines the transversal section of the leaf blade. The axis of this general cylinder is defined as the interpolated curve at time point $t$. The resulting sample frames of the animation are depicted on Figure 8.

\section{EXAMPLE OF FSPM APPLICATIONS IN L-Py}

In this section, we show how L-Py can be used to create complex FSPM scenarios. A first example illustrates how advanced analysis tools can be used to parameterize a L-system that reconstructs trees from observed data. The second example illustrates how modularity can be used to decompose an existing FSPM into reusable components. A last example reports the use of L-Py as a training tool for high school students to reconstruct a virtual ecosystem.

\section{MINIMIZING MEASUREMENTS IN 3D PLANT ARCHITECTURE RECONSTRUCTION}

The compatibility between L-Py and MTGs opens powerful new possibilities to manipulate plant simulations. Let us consider for example the problem of digitizing complex tree architectures. Different techniques to address this problem have been proposed in the literature and only manual techniques, such as magnetic $3 \mathrm{D}$ digitizing (Sinoquet et al., 1997), can currently precisely record both the 3D spatial coordinates and the topological structure of a plant in terms of annual shoots or growth units. Unfortunately, manual digitizing techniques are extremely time consuming and methods to simplify them are much needed. An intuitive idea is to exploit the redundancy of tree structures and only digitize the main branches of the tree. Smaller branchlets, which are highly repetitive, are then generated procedurally. Assuming that such a scheme has been implemented, the question is how to assess the resulting semi-automatic reconstruction method. The seamless combination of plant architecture simulation and analysis provided in L-Py makes it possible to simply address this issue.

Let us illustrate how such an approach would be implemented using L-Py. We assume that a reference plant has been digitized. Here, for sake of simplicity, we reuse the digitized walnut tree introduced previously. We also assume that a simple L-Py probabilistic model has been designed to generate small branches from bud modules. We want to assess the ability of this model to reconstruct faithfully the digitized small branches of the tree, and thus to avoid the overhead of digitizing small branches in similar trees.

For this, we first remove the small branching systems from the digitized tree. Modules of the digitized tree have three different types: modules of type $\mathrm{V}$ (resp. U) represent growth unit segments from the last year (resp. from the second last year). All other modules are of type $S$ and represent branch segments from previous years (Figure 9, left). In our example, we chose to remove the branching systems made up by growth units from the last 2 years, i.e., of type $\mathrm{V}$ and $\mathrm{U}$. This can be done by defining a simple L-Py rule in the previous L-system file (lsystem5.lpy) that replaces every branching system starting with a $\mathrm{U}$ and following a segment $\mathrm{S}$ by a bud:

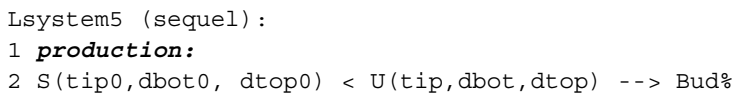

This rule removes in a single derivation step all the branch extremities starting with a $U$ from the multiscale $L$-string representing the digitized plant and replaces them by Bud modules (Figure 9, right).

In a subsequent derivation step, the bud modules are then used to produce new branching systems using a probabilistic model. This model is defined by an L-Py rule:

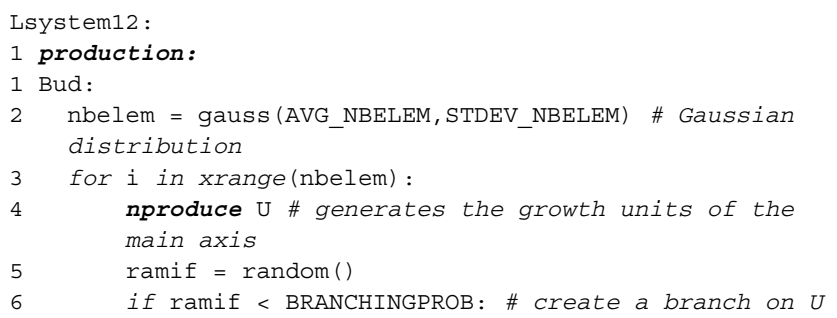




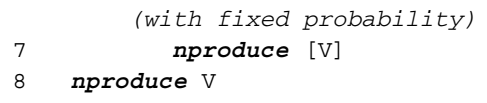

This rule specifies that a bud is replaced by a shoot made of a randomly chosen number of segments $U$ that can each bear (or not) a segment $\mathrm{V}$ and that terminate by a segment $\mathrm{V}$. Note that we omitted to show the computation of the branch geometry to keep the example simple. As a result, all the removed digitized branches are replaced by artificially generated small branches, thus providing a partially digitized and partially simulated tree.

Then, to assess the quality of the resulting semi-simulated tree, we make use of the plant structural comparison primitive available in the VPlants package of OpenAlea (Ferraro and Godin, 2000). This function compares the structures of two plants (here the digitized and semi-simulated trees) and returns a list of pairs

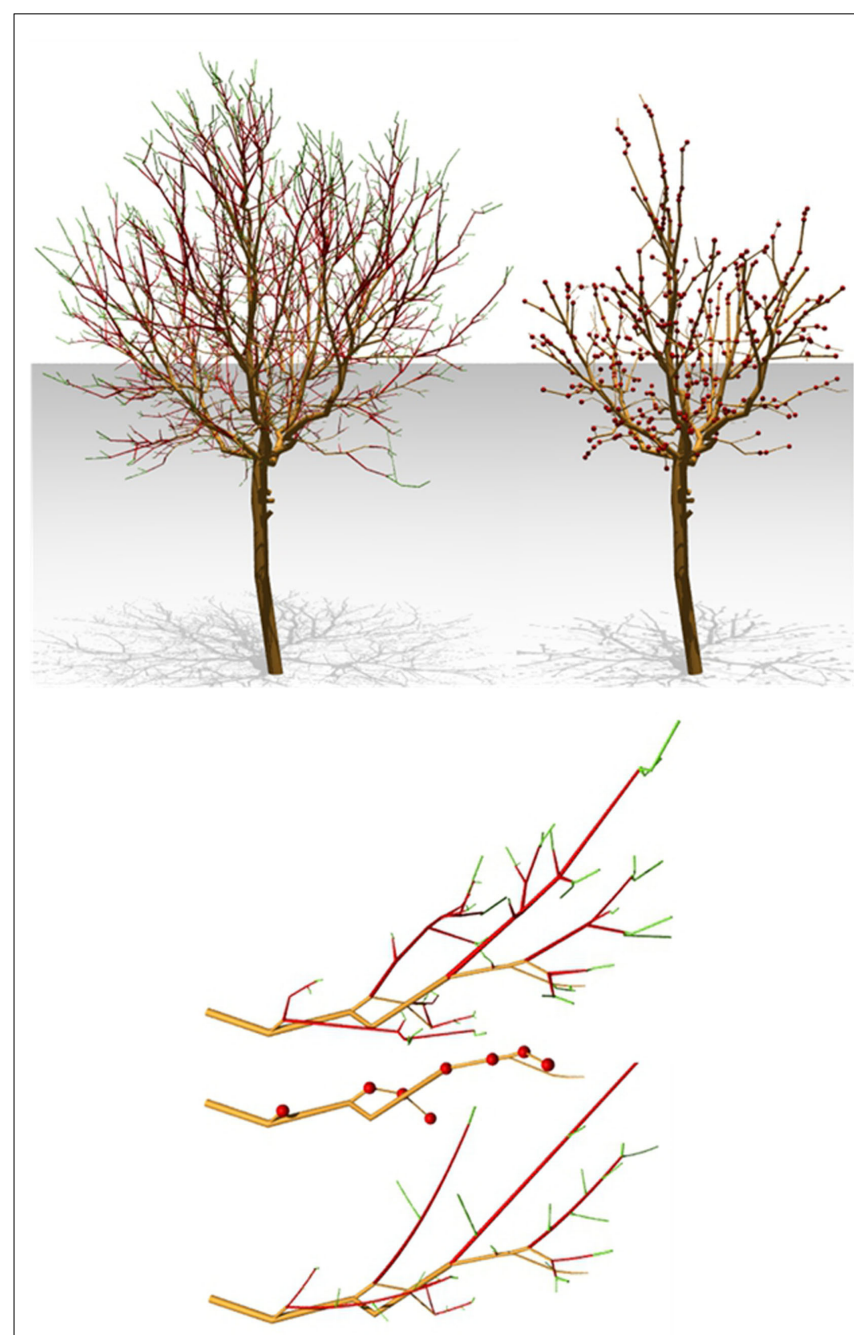

FIGURE 9 | Use of a digitized 20-year-old walnut tree (Juglans regia L.) as the axiom of a simulation. Right, shoots produced during the last 2 years are removed. Simulation process will use this structure as new axiom and produce algorithmically new shoots. Generated shoots will be compared with measured ones. Bottom, a detail view of the process: from top to bottom, original branching system, pruned system with insertion of Bud represented as red sphere, and example of regenerated structure. of plant segments from both plants that were found to match each other. The more matching segments are found in both trees, the better the reconstruction. The normalized length of the returned list, $Q=2 \times L /\left(L_{1}+L_{2}\right), L$ being the size of the returned list and $L_{1}$ and $L_{2}$ respectively the sizes of the compared trees, can thus be used as an indicator of the faithfulness of the model. If $Q$ is greater than a specified threshold, the simulated tree is considered as a faithful reconstruction and the list contains pairs referring to most of the components of both plants. In the opposite case, the list is close to being empty and the reconstruction is considered poor. The following function illustrates how such a comparison can be carried out in L-Py:

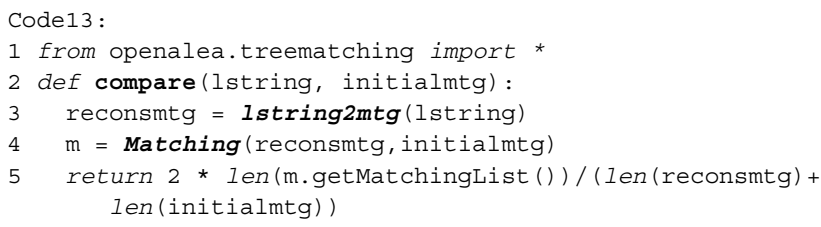

The compare function takes as arguments the current L-string representing the reconstructed plant and an MTG representing the initial digitized tree. It first transforms the L-string into an MTG and then compares the two MTG structures using a primitive from the treematching module of OpenAlea. As a result, it returns the estimated value of $Q$ for this comparison.

This function can then be used to explore the parameter space of the probabilistic branching model (here through varying the branching probability) so as to find those parameters that make it possible to reconstruct trees faithfully with respect to the original digitized tree. This is done in the following function that assembles the different components of this pipeline:
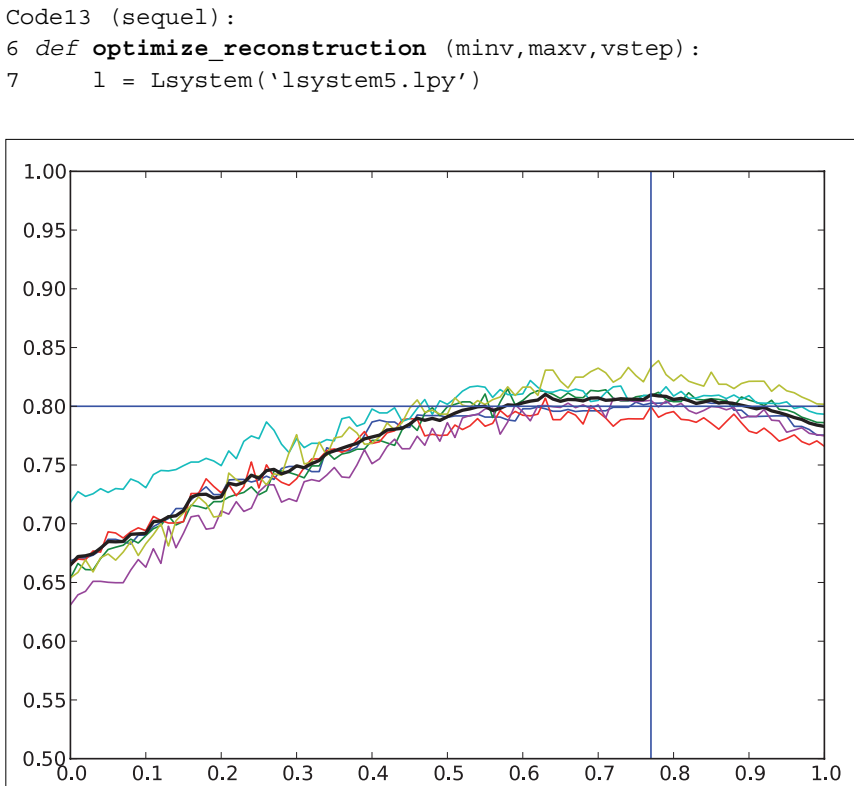

FIGURE 10 | Result of the comparison between regenerated structures and the measured one. The scores of the different branching systems are given by the colored curves and the average value by the black curve. Maximum average score is reached with a probability of 0.77 and gives a score of 0.8 


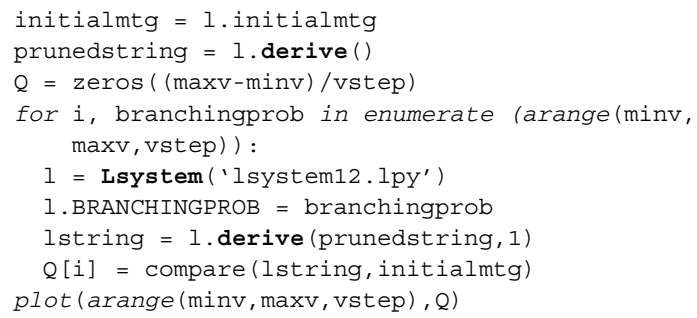

In our example, $Q$ varies non-monotonically between 0.65 and 0.85 when varying the probability parameter between 0 and 1 (see Figure 10), showing that best reconstructions are reached for a branching probability close to 0.77 in our stochastic model. Three examples of evaluated reconstruction are given in Figure 11 with branching probability of $0.4,0.6$, and 0.77 respectively. Red color represents parts of the structure whose $Q$ coefficient is greater than 0.8 .

\section{L-Py AS GROWTH COMPONENT FOR SIMULATING AN FSPM}

We now illustrate the use of the above modular approach on a real complex FSPM, MAppleT, simulating the growth of an apple tree (Costes et al., 2008) and originally developed using $L$ studio/lpfg. This model mixes stochastic topological construction with a bio-mechanical model for the geometry (see Figure 12). Thanks to syntax compatibility between L-Py and $L+C$, the code port mainly consisted in translating and simplifying the $\mathrm{C}++$ instructions into Python. Additionally, scientific tools from Python and OpenAlea were readily accessible from within the model (for instance, 2D plot with Matplotlib).

An L-system model, such as MAppleT, is composed of several processes that simulate the growth and internal processes of a plant. In the original model, groups of rules were defined to model different processes: (a) updating state of organs according to a calendar (bud break, flowering, etc.), (b) computation of growth units lateral productions according to stochastic

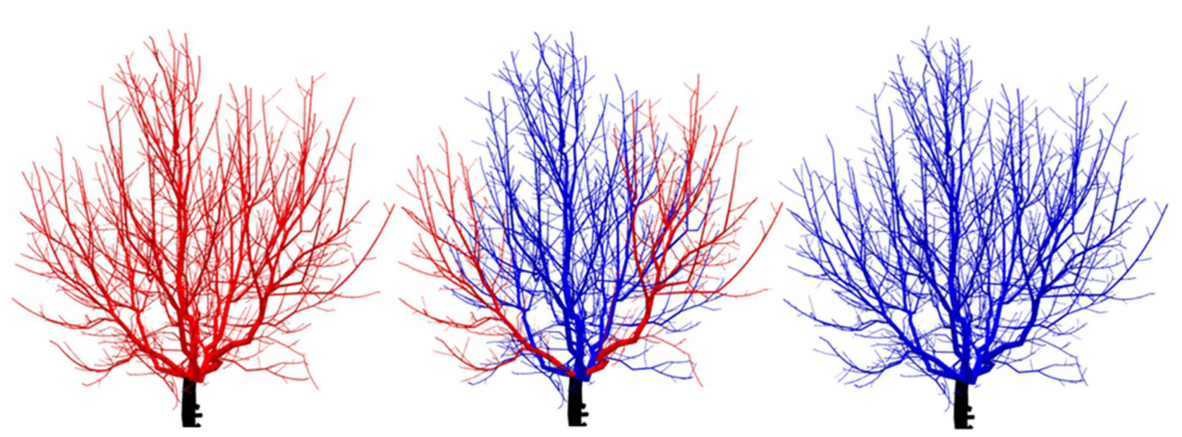

FIGURE 11 | Comparison between regenerated structure and measured one. Reconstructions showed here are built with ramification probability of $0.4,0.6$, and 0.8 respectively. The six main branching systems of the tree are compared to the original ones using a structural comparison method (Ferraro and Godin, 2000). Red and blue color means that structural difference is more or less than $20 \%$ respectively.

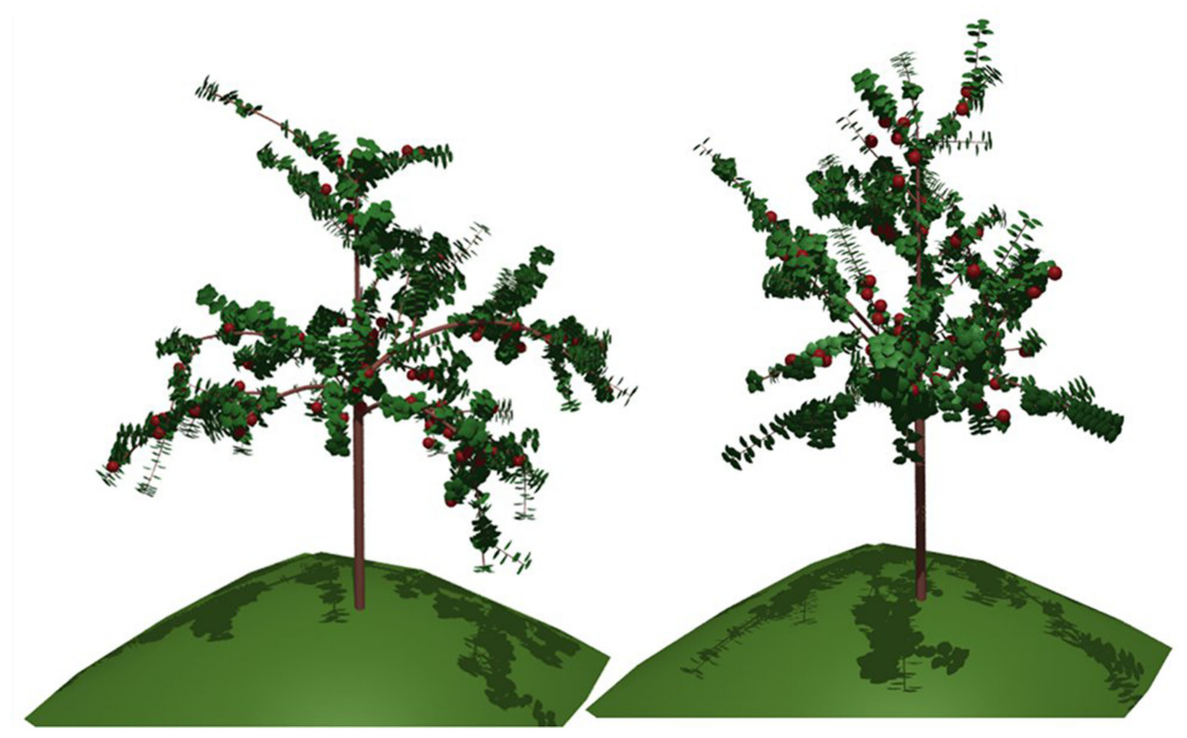

FIGURE 12 |The MAppleT model on L-Py: with (left) and without (right) computation of branch bending using mechanical simulation. 
models, (c) growth process, (d) biomechanics. During refactoring, the code was divided into distinct L-systems corresponding to these different groups of rule to achieve modularity. Because they came from a single original L-system code, these L-systems components used similar module naming convention and thus were readily compatible with each other (see Modular L-Systems in L-Py). The parameters of the modules were stored in a generic container object whose contents can be updated by each L-system component. Parameters used by different Lsystem components were given a unique name in all of these. For instance, the growth process component (c) requires information on the number and types of components to create at each time step. This information is provided by the stochastic process component (b) using a consistent naming convention in both components.

Usually the different processes also rely on a number of global variables. To change their values, a dictionary containing the names and values of global variables can be passed to the L-systems and can be applied using the introspection mechanism presented in Section "L-Py as a Component Library: Controlling L-Systems Execution from Python." In this way, the global variables can be passed from one process to the next one. Each process can thus update these settings to inform the other processes if needed.

To better demonstrate the modularity of the code resulting from this decomposition of MAppleT, the L-system components were assembled graphically using a dataflow in OpenAlea (Figure 13). As opposed to code representation, dataflows give a visual representation of the logical dependency structure of the FSPM. The composition of the components can be made graphically by the modeler by linking input and output of the different L-systems components and making it possible for the system to pass on the L-string and the dictionary of global parameters. The resulting graph (dataflow) can be executed and runs the pipeline throughout.

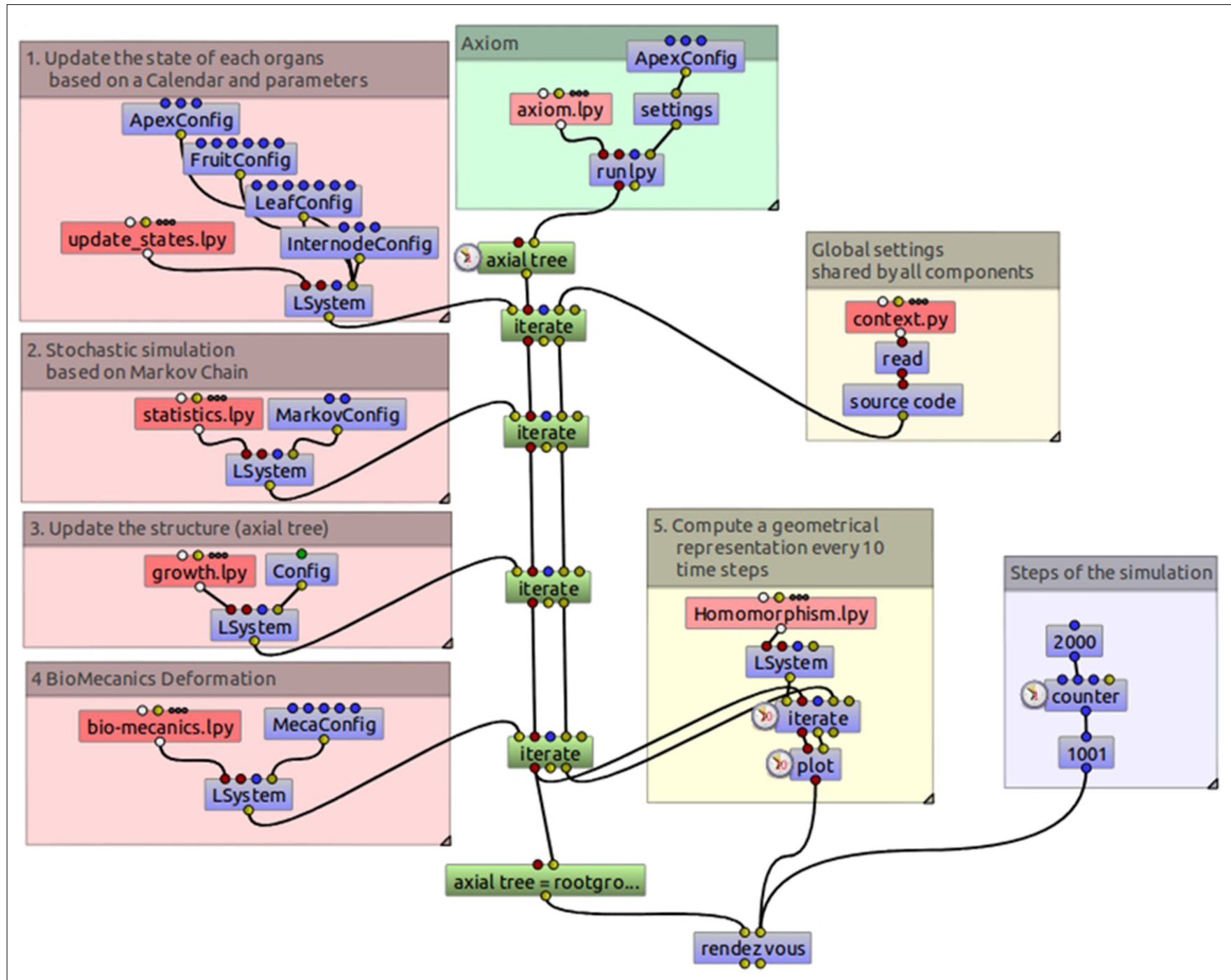

FIGURE 13 | Data flow of the MAppleT simulation. The model has been decomposed into several independent processes that can be combined and parameterized by user to drive the simulation graphically. 
Thanks to this modular decomposition, interesting manipulation of the assembled models can be made. For example, the user has the possibility to enable/disable some of them upon request. Figure 12 right illustrates for example the result of the model in which the Biomechanics component has been disabled.

Based on this implementation of a real-size FSPM, we could carry out a comparative analysis of the computational performances of L-Py and that of static language implementations. L-Py was able to generate an entire architecture (10,000 components generated over 4 years) in reasonable time (5-10 $\mathrm{min})$. In general, simulation with L-Py can be faster than with $l p f g$ for small models (since it avoids compilation), but is five to six time slower than $l p f g$ for more complex models of a 4 -year-old apple tree (see

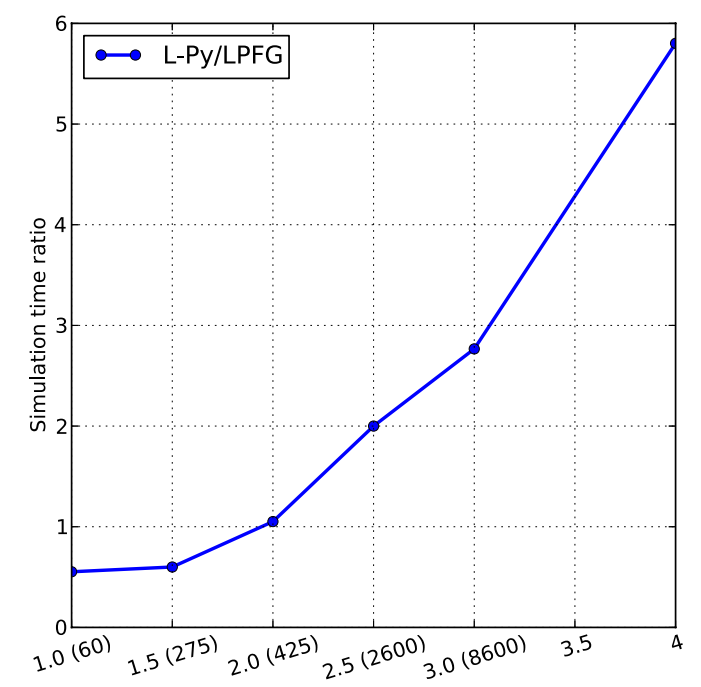

FIGURE 14 | Computation time comparison with between L-Py and Ipfg for MAppleT. The horizontal axis represents age of the simulated tree with estimated number of elements of the tree.
Figure 14). This is due to Python code interpretation of rules which is relatively slow compared to a compiled language like $\mathrm{C}$ or C++ (Prechelt, 2000). However, the L-Py interpreter written in $\mathrm{C}++$ maintains acceptable performances.

\section{L-Py AS A TRAINING TOOL FOR THE CLASSROOM}

During French school year 2009-2010, we tested the use of L$\mathrm{Py}$ as a tool for teaching scientific method in the context of a multi-disciplinary class on botany and computer science at highschool level (15- to 16-years-old pupils, $3 \mathrm{~h}$ per week during 35 weeks). The program of the class included both botanical and computer science/mathematics courses. The aim of the class was to reconstruct in $3 \mathrm{D}$ the vegetal structure of a $10 \mathrm{~m} \times 10 \mathrm{~m}$ plot of plants typical from the local flora. The pupils measured the plants in the field, made diagrams and drawings of the plant architectures (see Figure 15), and registered the spatial distribution of observed plants. In the classroom, they were working hands on the computer and using L-Py as a modeling platform. They first learnt how to generate simple fractal and plant structures. They could create soon first simple models of plant structures. Then, using more sophisticated and generic models prepared for the occasion, they easily used their knowledge of L-Py to extend and customize these models according to the measured plants. Modifications ranged from simple parameter modification to addition of new rules in the L-systems. A number of individual plant models were thus designed by different groups of pupils in L-Py and were assembled into a single scene according to the measured distribution. Finally the scene was exported and rendered with Blender (2011) and a film corresponding to a virtual exploration of the 3D scene was produced. This experience gave us important feedback on L-Py during its testing phase. It first showed that the software can be used with success for training students in a multi-disciplinary context. L-Py turned out to be robust enough to support intensive use (and misuse) by pupils. The feedback from the classroom led us to adapt L-Py in various ways: simplify the visual interface, introduce debugging tools, and design new language features (such as the SetGuide and the curve interpolation primitives).
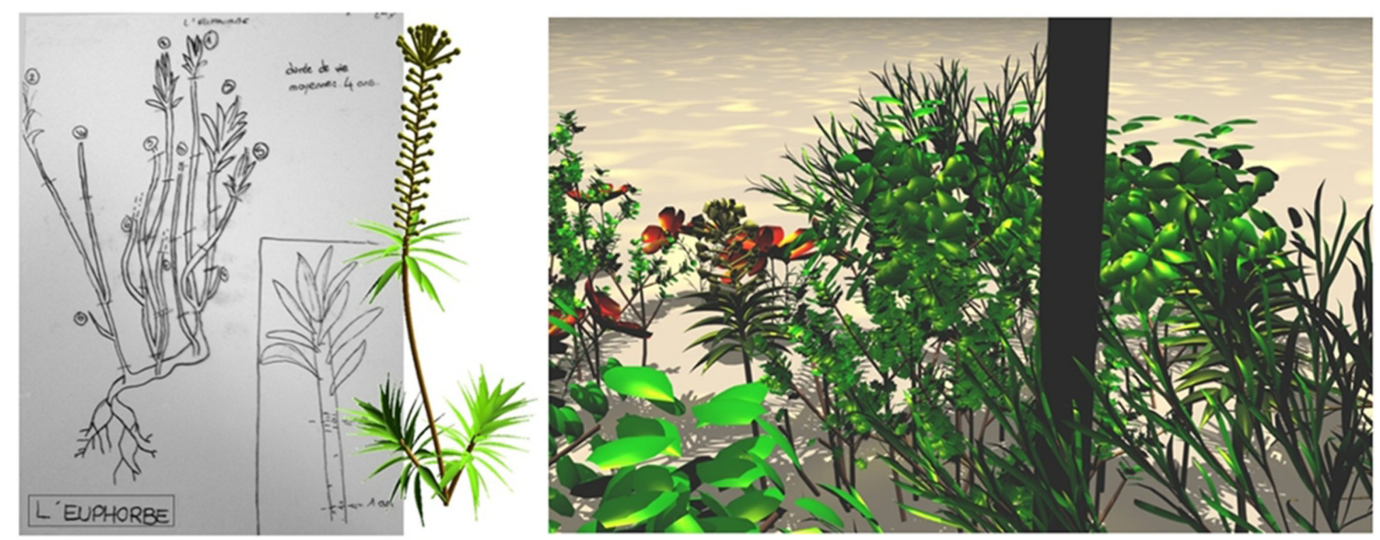

FIGURE 15 | Illustration of an application of L-Py in teaching. Students were in the field to map some botanical drawing of Euphorbia. Some virtual models were then build with L-Py and gathered in a complex botanical scene rendered with Blender. 


\section{CONCLUSION}

In this paper, we presented L-Py, an open-source software platform for L-system simulation based on Python. Compared to previous L-system simulation software packages, L-Py makes intensive use of Python's property of being a dynamic language to achieve flexibility in modeling and high-level programming. Inherited from Python, the L-Py language syntax remains simple, with no or minimal bracketing of expressions, and very clear block structures. L-Py was designed as both an integrative framework and a library of L-system constructs that can be called from Python. Owing to its dynamic language structure, L-Py facilitates the design of modular models by assembling elementary models without modifying their code, provided they respect minimal specifications. For the design of plant models, special language constructs were introduced for compliance with MTG data-structures, for which large libraries of computational models and tools already exist (e.g., OpenAlea software platform and its packages). Likewise, for geometry, constructs were introduced to reuse any model from PlantGL,

\section{REFERENCES}

Barthélémy, D., and Caraglio, Y. (2007). Plant architecture: a dynamic, multilevel and comprehensive approach to plant form, structure and ontogeny. Ann. Bot. 99, 375-407.

Bell, A. D. (1991). Plant Form: An Illustrated Guide to Flowering Plant Morphology. Oxford: Oxford University Press.

Blender. (2011). The Blender Foundation. Amsterdam. Available at: http://www.blender.org

Bloomenthal, J. (1985). "Modeling the mighty maple," in Proceedings of the 12th Annual Conference on Computer Graphics and Interactive Techniques, SIGGRAPH '85 (New York: ACM).

Bloomenthal, J. (1990). "Calculation of reference frames along a space curve," in Graphics Gems, ed A. S. Glassner (Boston: Academic Press), 567-571.

Boudon, F. (2004). Représentation Géométrique de L'architecture des Plantes. Ph.D. thesis, University of Montpellier II, Montpellier.

Cescatti, A. (1997). Modelling the radiative transfer in discontinuous canopies of asymmetric crowns. I. Model structure and algorithms. Ecol. Modell. 101, 263-274.

Cieslak, M., Seleznyova, A. N., Prusinkiewicz, P., and Hanan, J. (2011). Towards aspect-oriented functionalstructural plant modelling. Ann. Bot. 108, 1025-1041.

Costes, E., Smith, C., Renton, M., Guédon, Y., Prusinkiewicz, P., and Godin, C. (2008). MAppleT: simulation of apple tree development using mixed stochastic and biomechanical models. Funct. Plant Biol. 35, 936-950.
Da Silva, D., Boudon, F., Godin, C. and Sinoquet, H. (2008). Multiscale framework for modeling and analyzing light interception by trees. Multiscale Model. Simul. 7, 910-933.

Federl, P., and Prusinkiewicz, P. (1999). "Virtual laboratory: an interactive software environment for computer graphics," in Proceedings of Computer Graphics International, Canmore: Alberta. '99, 93-100.

Federl, P., and Prusinkiewicz, P. (2004). "Solving differential equations in developmental models of multicellular structures expressed using L-systems," in Proceedings of Computational Science. ICCS 2004 (Krakow, Poland, June 6-9, 2004), Part II, Lecture Notes in Computer Science 3037, eds M. Bubak, G. van Albada, P. Sloot, and J. Dongarra (Berlin: Springer), 65-72.

Ferraro, P., and Godin, C. (2000). A distance measure between plant architecture. Ann. For. Sci. 57, 445-461.

FSPM Special Issue. (2005). New Phytologist. Blackwell Publishing. 166, 771-894.

FSPM Special Issue. (2008). Functional Plant Biology. Eds J. Hanan and P. Prusinkiewicz, CSIRO Publishing, 35, 739-1090.

FSPM Special Issue. (2011). Annals of Botany. Eds T. de Jong and D. Da Silva (Oxford: Oxford University), 108, 987-1223,

Godin, C., and Caraglio, Y. (1998). A multiscale model of plant topological structures. J. Theor. Biol. 191, $1-46$.

Godin, C., Costes, C., and Caraglio, Y. (1998). Exploring plant topological structure with the AMAPmod

a library of geometric primitives dedicated to plant representation at different scales, and to simplify the specification of complex geometric models with the L-system turtle. To illustrate the flexibility and power of L-Py, we presented its application to real modeling situations. The problem of optimizing plant digitizing strategy illustrated the benefit of using jointly L-systems and MTGs in a modeling application. MappleT was used to illustrate the possibility to design complex simulation systems in a modular way. The last example illustrated the pedagogic value of L-Py based on a real experiment carried out with high-school students in France.

\section{ACKNOWLEDGMENTS}

The authors are grateful to M. Beziz, E. Farcot, Y. Caraglio, D. Lacour, L. Comte, and J. Chopard who were involved in the high-school classes. This project is partially supported by Agropolis Foundation and the INRIA VPlants-BMV associated team project.

software; an outline. Silva Fennica 31, 357-368.

Godin, C., Costes, E., and Sinoquet, H. (1999). A method for describing plant architecture which integrates topology and geometry. Ann. Bot. 84, 343-357.

Godin, C., Costes, E., and Sinoquet, H. (2005). "Plant architecture modelling - virtual plants and complex systems," in Plant Architecture and its Manipulation, Vol. 17, ed C. G. N. Turnbull (Oxford: Blackwell) 238-287.

Godin, C., and Guédon, Y. (1997). AMAPmod v1.8. Introduction and Reference Manual. CIRAD Report, Montpellier.

Godin, C., and Sinoquet, H. (2005). Functional-structural plant modelling. New Phytol. 166, 705-708.

Hallé, F. (1978). "Architectural variation at specific level of tropical trees," in Tropical Trees as Living Systems, eds P. B. Tomlinson and M. H. Zimmermann (Cambridge: Cambridge University Press) 209-221.

Hanan, J. (1992). Parametric L-Systems and their Application to the Modeling and Visualization of Plants. Ph.D. dissertation, University of Regina, Regina, Saskatchewan.

Hanan, J., and Prusinkiewicz, P. (2008) Foreword: studying plants with functional-structural models. Funct. Plant Biol. 35, vi-viii.

Herman, G. T., and Rozenberg, G. (1975). Developmental systems and languages. North-Holland: Amsterdam.

Karwowski, R., and Prusinkiewicz, P. (2003). Design and implementation of the $\mathrm{L}+\mathrm{C}$ modeling language.
Electron. Notes Theor. Comput. Sci. 86, 134-152.

Kniemeyer, O., and Kurth, W. (2008). "The modelling platform GroIMP and the programming language $\mathrm{XL}$," in Applications of Graph Transformations with Industrial Relevance: Third International Symposium, AGTIVE 2007, Kassel, Germany, October 10-12, 2007, Revised Selected and Invited Papers, Lecture Notes In Computer Science, 5088, eds A. Schürr, M. Nagl, and A. Zündorf (Kassel: Springer-Verlag), 570-572.

Lindenmayer, A. (1968). Mathematical models for cellular interaction in development, Parts I and II. J. Theor. Biol. 18, 280-315.

Livny, Y., Pirk, S., Cheng, Z., Yan, F., Deussen, O., Cohen-Or, D., and Chen, B. (2011). Texture-lobes for tree modelling. ACM Trans. Graph. $30,53$.

Mercer, L., Prusinkiewicz, P., and Hanan, J. (1990). "The concept and design of a virtual laboratory," in Proceedings of Graphics Interface. Halifax, Nova Scotia. '90, 149-155.

Microsoft Corporation. (2011). The Visual Studio. Available at: http:// www.microsoft.com/visualstudio/

Mündermann, L., Erasmus, Y., Lane, B., Coen, E., and Prusinkiewicz, P. (2005). Quantitative modeling of Arabidopsis development. Plant Physiol. 139, 960-968.

Ousterhout, J. K. (1998). Scripting: higher-level programming for the 21st century. IEEE Comput. 31, 23-30.

Piegl, L. A., and Tiller, W. (1997). The Nurbs Book, 2nd Edn. Berlin: Springer. 
Pradal, C., Boudon, F., Nouguier, C., Chopard, J., and Godin, C. (2009). PlantGL: a Python-based geometric library for 3D plant modelling at different scales. Graph. Models 71, $1-21$.

Pradal, C., Dufour-Kowalski, S., Boudon, F., Fournier, C., and Godin, C. (2008). OpenAlea: a visual programming and componentbased software platform for plant modeling. Funct. Plant Biol. 35, 751-760.

Prechelt, L. (2000). An empirical comparison of seven programming languages. IEEE Comput. 33, 23-29.

Prusinkiewicz，P. (1986). "Graphical applications of L-systems," in Proceedings of Graphics Interface '86, 247-253.

Prusinkiewicz, P. (1998). Modeling of spatial structure and development of plants. Sci. Hortic. 74, 113-149.

Prusinkiewicz, P. (1999). A look at the visual modeling of plants using Lsystems. Agron. Agric. Environ. 19, 211-224.

Prusinkiewicz, P. (2004). "Art and science for life: designing and growing virtual plants with L-systems," in Nursery Crops: Development, Evaluation, Production and Use: Proceedings of the XXVI International Horticultural Congress, Acta Horticulturae
630, eds C. Davidson and T. Fernandez, (ISHS: Toronto), 15-28.

Prusinkiewicz, P., Hanan, J., and Mech, R. (1999a). "An L-system-based plant modeling language," in Applications of Graph Transformations with Industrial Relevance. Proceedings of the International Workshop AGTIVE '99, Lecture Notes in Computer Science 1779, eds M. Nagl, A. Schuerr, and M. Muench (Berlin: Springer), 395-410.

Prusinkiewicz, P., Karwowski, R., Mech, R., and Hanan, J. (1999b). "Lstudio/cpfg: A software system for modeling plants," in Applications of Graph Transformations with Industrial Relevance. Proceedings of the International Workshop AGTIVE '99, Lecture Notes in Computer Science 1779, eds M. Nagl, A. Schuerr, and M. Muench (Berlin: Springer), 457-464. Prusinkiewicz, P., James, M., and Mech, R. (1994). "Synthetic topiary," in Proceedings of SIGGRAPH Orlando, Florida. '94, 351-358.

Prusinkiewicz, P., Karwowski, R., and Lane, B. (2007). "The L+C plant modeling language," in FunctionalStructural Plant Modeling in Crop Production, eds. J. Vos, L. F. M. Marcelis, P. H. B. de Visser, P. C. Struik, J. B. Evers and R. J. Bogers, (Wageningen: Springer), 27-42.
Prusinkiewicz, P., and Lindenmayer, A. (1990). The Algorithmic Beauty of Plants. New York: Springer.

Prusinkiewicz, P., Lindenmayer, A., and Hanan, J. (1988). Developmental models of herbaceous plants for computer imagery purposes. Comput. Graph. 22, 141-150.

Prusinkiewicz, P., Mündermann, L., Karwowski, R., and Lane, B. (2001). "The use of positional information in the modeling of plants," in Proceedings of SIGGRAPH '2001, Los Angeles, 289-300

Sievänen, R., Mäkelä, A., Nikinmaa, E., and Korpilahti, E. (1997), Special issue on functional-structura tree models. Silva Fennica 31, 237-238.

Sinoquet, H., Rivet, P., and Godin, C. (1997). Assessment of the threedimensional architecture of walnut trees using digitising. Silva Fennica 31, 265-273.

Teobaldelli, M., Puig, A. D., Zenone, T., Matteucci, M., Seufert, G., and Sequeira, V. (2008). Building a topological and geometrical model of poplar tree using portable onground scanning LIDAR. Funct. Plant Biol. 35, 1080-1090.

Tratt, L. (2009). Dynamically typed languages. Adv. Comput. 77, 149-184.
Wang, W., Jüttler, B., Zheng, D., and Liu, Y. (2008). Computation of rotation minimizing frame. ACM Trans. Graph. 27, 18.

Conflict of Interest Statement: The authors declare that the research was conducted in the absence of any commercial or financial relationships that could be construed as a potential conflict of interest.

Received: 16 December 2011; accepted: 04 April 2012; published online: 30 May 2012.

Citation: Boudon F, Pradal C, Cokelaer T, Prusinkiewicz $P$ and Godin $C$ (2012) L-Py: an L-system simulation framework for modeling plant architecture development based on a dynamic language. Front. Plant Sci. 3:76. doi: 10.3389/fpls.2012.00076

This article was submitted to Frontiers in Technical Advances in Plant Science, a specialty of Frontiers in Plant Science. Copyright () 2012 Boudon, Pradal, Cokelaer, Prusinkiewicz and Godin. This is an open-access article distributed under the terms of the Creative Commons Attribution Non Commercial License, which permits non-commercial use, distribution and reproduction in other forums, provided the original authors and source are credited. 


\section{APPENDIX \\ STANDARD L-SYSTEMS FEATURES OF L-Py}

L-Py supports a number of standard features introduced in other L-system-based programming languages. As illustrated by the first example of this paper, L-Py supports parametric L-systems with flexible parameter types. Conditional rule application is thus possible. By default, rules are deterministic. However, stochastic rules are quite natural to build using functions from statistical packages provided by Python. A typical example would be

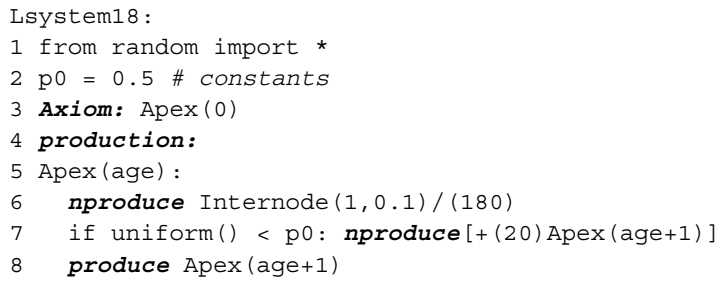

The first line imports the Python package random for random number generation. This package includes the uniform function used at line 8 . Line 2 defines branching probability 0 . The rule in lines 5-8 uses the nproduce statement to assemble the successor in several steps. In particular, the production of a lateral apex (line 8) depends on the value returned by the uniform random function, which is compared to $\mathrm{p} 0$.

Production rules make it possible to insert new module. Likewise, the disappearance of a module is specified using productions with an empty successor. An entire sub-tree can be removed by inserting the module \%, which prunes out all the following sub-structure in the string until the end of the enclosing branch.

Several aspects of a derivation step can be controlled by the user. First, a rule can be applied by scanning the string from left to right (forward), which corresponds to an acropetal progression from the root of the structure to the leaves, or from right to left (backward), i.e., basipetally from the leaves to the root (Karwowski and Prusinkiewicz, 2003). The functions Forward and Backward can be called to parameterize the direction of the next iteration. This is usually set up using the control functions Start, End, StartEach, and EndEach, which are defined by the user and called by the system at the beginning, end, before, and after a derivation step, respectively (Hanan, 1992). Second, it is possible to select a subset of rules at a given step. For this, a group of rules can be defined and activated during execution of the model to simulate separately different aspects of the behavior of a model (Prusinkiewicz et al., 2007).

Application of a rule may depend on the context of the predecessor using context-sensitive rules. L-Py also supports four types of context, namely left, right, new left, and new right contexts (Karwowski and Prusinkiewicz, 2003). Notation follows the $L+$ C syntax, i.e., the predecessor may have the following form for forward and backward derivation, respectively

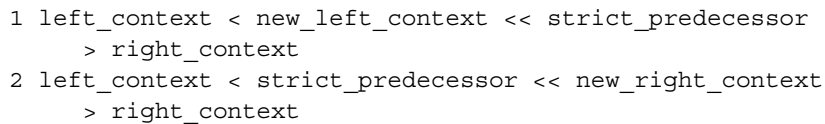

The context search takes into account the branching structure of trees. For instance the following pattern $\mathrm{A}<\mathrm{C}$ supposes a fatherchild relationship between module $A$ and $C$, but does not exclude other children of $A$. In particular, the string $w=A[B][C]$ represents a topology where a module $A$ has two children $B$ and $C$. The previous pattern thus applies to $\mathrm{C}$. Some modules within branches may thus be skipped during a linear scan of the tree. Contexts are particularly useful to simulate signals and communication between organs (Prusinkiewicz et al., 1988; Prusinkiewicz and Lindenmayer, 1990).

L-Py models may also be sensitive to their spatial location. For this, query modules, such as ? P, make it possible to get position and orientation of the geometrical interpretation of the model in the rewriting process (Prusinkiewicz et al., 1994).

\section{MANAGING L-SYSTEM MODULARITY}

To design modular simulation systems in L-Py, the user must comply with three simple rules when breaking down the system into different L-systems.

\section{Rule 1. Model consistency}

Assume that, at a given time $t$, all the L-systems will be applied to identical plant topological structures. Only names and attributes of plant components can be different.

\section{Rule 2. Single generic module parameter}

If different attributes must be attached to a particular module (e.g., age, diameter, etc.), they must be encapsulated within a single parameter that consists of an object of the built-in type Parameterset. The plant components attributes are then represented as attributes of this object.

For example, let us consider a model A that uses two types of modules namely Apex and Internode:

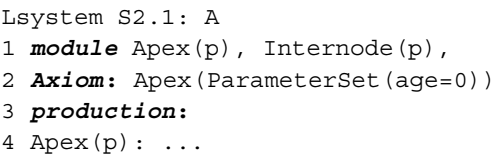

In the L-string, the modules have one parameter $\mathrm{p}$, which is a predefined generic container of type Parameterset. Each Lsystem rule can then dynamically add or modify attributes to this generic parameter p. Here, we again take advantage of the flexibility of dynamic language, in which a Python structure can be complemented with new attributes at any moment by any L-system without any explicit pre-declaration. This makes it possible for each L-system to add its own attributes to the generic parameter $\mathrm{p}$, without requiring that these attributes are know to other L-systems. This mechanism is clearly more flexible that of static languages in which parameter declaration is mandatory at the initialization and should be conform to this declaration in every part of the code, thus contaminating all the L-systems components.

Rule 3. Translation schemes. Each L-system may have different module naming convention and attributes. For a given L-system $B$ to be able to process the L-string of L-system A, it is necessary that a mapping from $\mathrm{A}$ modules to $\mathrm{B}$ modules be defined, thus acting as a translation from the language of $\mathrm{A}$ to the language of $\mathrm{B}$. Our approach consists of transforming A modules into B modules and 
back using L-system rules. Module name translation can easily be expressed with L-system rules as illustrated by the Lsystem A2B below that transforms A modules into corresponding B ones.

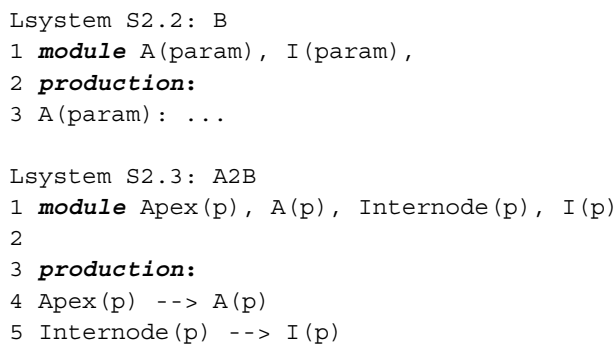

Symmetric rules may be required to translate back from the language of $\mathrm{B}$ into the language of $\mathrm{A}$, in particular if one wants to iteratively apply L-systems A, B several times consecutively. Such a bidirectional translation requires that mapping from the alphabet of $\mathrm{A}$ to the alphabet of $\mathrm{B}$ is one to one. This is done by defining a new L-system:

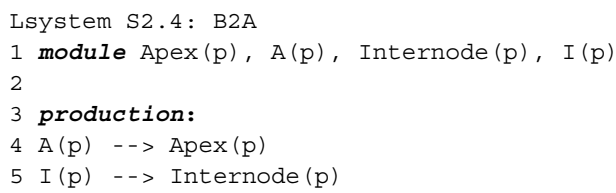

Similarly, attribute names used in the parameter container should be consistent between different L-systems. If, for instance, two processes rely on similar attributes but use different naming convention, attributes will also have to be renamed during translation step. Conversely, if two processes use the same attribute name for different things, attributes will also have to be renamed to avoid a conflict.

Then, at a given time step, starting from an A L-string, the user applies L-system A which computes a new A L-string. Then this A $\mathrm{L}$-string is translated into a B L-string with L-system B2A so that the L-system B can be applied. Then B is applied and finally, the resulting B string is translated back into A L-string to come back to the initial A L-system and continue the simulation. The procedure can be readily extended to the combination of any number of L-systems.

The L-systems can then be assembled into a super-L-system using the built-in ComposedLsystem primitive described in the main text.

\section{THE SetGuide PRIMITIVE}

A turtle is associated with a position $P$ and frame $F_{\mathrm{T}}$ composed of three orientation vectors mutually perpendicular that indicates its heading $H$, up $U$, and left directions $L$ (Prusinkiewicz et al., 2001). The turtle can perform a number of geometric operations. In particular, it can step forward in its heading direction or can rotate along its three orientation axis. Following a predefined path with turtle geometry was described in Prusinkiewicz et al. (2001) and consists of adjusting orientation of the turtle before each step to fit the heading vector to the local path tangent $T$.

Similarly, the SetGuide makes it possible that steps of a turtle follow the path of a user-defined curve $C$, scaled to reach a length
$L$ also specified by the user. The turtle steps are not supposed in this case to be small. The curve is supposed to be positioned and oriented in the initial location of the turtle. It is also supposed to be parameterized with curvilinear abscissa $u$.

To compute orientations along a curve, a frame can be computed at each point of $C$. A frame is made of three vector $(r, s$, $t$ ) with $t$ representing the tangent to the curve. Classical solution relies on the Frenet frame. However, it produces important twist of the frame at inflection point of the curve. Some methods make it possible to compute frame with minimized rotation (Bloomenthal, 1990; Wang et al., 2008). In our approach, we adapt the method described in Prusinkiewicz et al. (2001). It starts from a frame $F(r, s, t)$ at $C(u)$. To compute $F^{\prime}\left(r^{\prime}, s^{\prime}, t^{\prime}\right)$ at $C(u+\mathrm{du})$, the tangent $t^{\prime}$ of the curve is first estimated. Then the rotation $R$ that maps $t$ to $t^{\prime}$ is computed. This rotation can then be applied to $s$ and $r$ of $F$ to have $r^{\prime}$ and $s^{\prime}$ values. The advantage of this method is that it is consistent (no twist) and iterative with only the value of the previous step needed to compute the next one.

To follow the path of the curve, rotations of the turtle will be performed before and after each step to orient it in a direction given by the curve $C$. The step forward command $(F)$ with a parameter du representing step length is thus decomposed into three steps: adjust orientation to reach $C(u+\mathrm{du})$, move the turtle forward, and adjust the orientation of the turtle to fit tangent of $C$ at $u+$ du (see Figure A1). The first and last operations are turtle orientation performed using the method presented previously. In the first case, the new tangent direction is approximated with the vector $d=C(u+\mathrm{du})-C(u)$.

One problem comes from the fact that the rotation is computed in the coordinate system of the curve. To adapt it for the turtle frame, the axis $A$ of rotation $R$ is translated from the reference frame $F(u)$ of the curve to the frame $F_{\mathrm{T}}$ of the turtle: $A^{\prime}=F_{\mathrm{T}} \times F(u)^{-1} \times A$. The transformation $R^{\prime}\left(A^{\prime}, \alpha\right)$ can then be applied to the vectors $H, L$, and $U$ of the turtle frame to express the reorientation of the turtle. Note that $R(A, \alpha)$ is still used to computed the new frame of the curve at $C(u+\mathrm{du})$. Finally when moving the turtle forward, the length of the step has to be adapted.

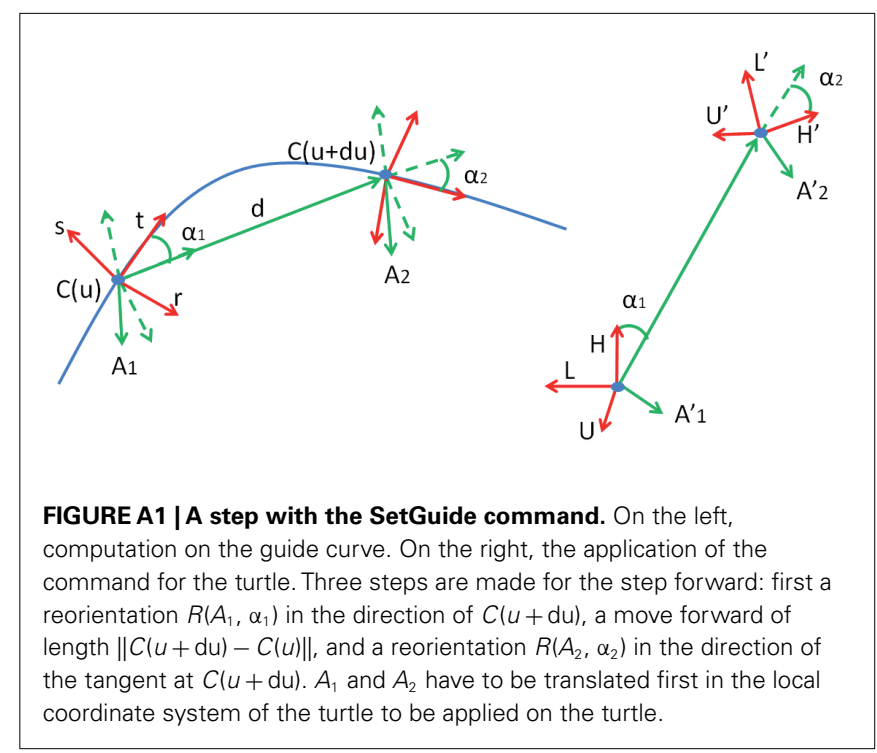


Indeed, the length of the curve between $C(u)$ and $C(u+\mathrm{du})$ is du but the curve between this two points is approximated with a straight segment by the turtle. Thus the length of turtle step should be changed for $\|C(u+\mathrm{du})-C(u)\|$.

The turtle will thus produce a path with curvature similar to the curve $C$ when moving forward. Additionally, some rotations can also be explicitly asked by the user to the turtle between each step. By default, these rotations will provide additional curvature to the turtle path. For instance, trunk and main branches can be built using the same SetGuide command except that at the beginning of branches an additional rotation is made to have lateral direction. In this case, guide and turtle are rigidly linked since the rotation of the turtle will also change the guide curve reference frame.

It is also possible to apply similar rotations of the turtle to the frame along the guide. For instance, the Roll operation consists in turning around the heading $H$ of the turtle. Its counterpart consists in turning the reference frame $F(r, s, t)$ of the curve $C$ at $C(u)$ around its tangent $t$. Both operations can be made at the same time when turtle is requested to turn. In this case, guide and turtle are non-rigidly linked since a turtle rotation will change the corresponding position and orientation on the curve $C$. Such operations are made possible in L-Py with special command $i R O l l R$ and iRollL. 